\title{
ABOUT STABILITY AND REGULARIZATION OF ILL-POSED ELLIPTIC CAUCHY PROBLEMS: THE CASE OF $C^{1,1}$ DOMAINS
}

\author{
LAURENT BOURGEOIS ${ }^{1}$
}

\begin{abstract}
This paper is devoted to a conditional stability estimate related to the ill-posed Cauchy problems for the Laplace's equation in domains with $C^{1,1}$ boundary. It is an extension of an earlier result of [Phung, ESAIM: COCV $\mathbf{9}$ (2003) 621-635] for domains of class $C^{\infty}$. Our estimate is established by using a Carleman estimate near the boundary in which the exponential weight depends on the distance function to the boundary. Furthermore, we prove that this stability estimate is nearly optimal and induces a nearly optimal convergence rate for the method of quasi-reversibility introduced in [Lattès and Lions, Dunod (1967)] to solve the ill-posed Cauchy problems.
\end{abstract}

Mathematics Subject Classification. 35A15, 35N25, 35R25, 35R30.

Received November 12, 2008. Revised June 11, 2009 and September 9, 2009.

Published online February 23, 2010.

\section{INTRODUCTION}

The question of stability for ill-posed elliptic Cauchy problems is a central question in the fields of inverse problems and controllability. Concerning the inverse problems for example, the aim is generally to retrieve some unknown object, for example an obstacle or a distributed parameter, with the help of some boundary measurements. These measurements are noisy data by nature. Given two sets of data the distance of which is $\sigma$, the problem of stability amounts to study the distance in term of $\sigma$ between the corresponding two retrieved objects. In particular, a practical motivation is numerics: the better is the stability we obtain, the better is the numerical reconstruction we expect. In this view, the stability for ill-posed elliptic Cauchy problems is an important first step in order to study the stability of more complex inverse problems governed by elliptic PDEs, as it may be seen for example in [1] concerning the inverse obstacle problem and in [6] concerning the corrosion detection problem. The following paper is focused on this first step.

A number of authors have studied the stability for ill-posed elliptic Cauchy problems since the first contributions of John [14] and Payne [20]. In the meantime, the so-called Carleman estimates have become a very efficient tool to derive not only unique continuation properties (see for instance [5,22]) but also stability estimates (see for instance $[6,13,18,21,24]$ ).

The obtained stability estimates take some various forms, depending on the geometry of the domain and on the regularity of the function. But a classical and general result is that the stability estimates are, following the vocabulary introduced by John [14], of Hölder type in a subdomain which does not include a neighborhood

Keywords and phrases. Carleman estimate, distance function, elliptic Cauchy problems, conditional stability, quasi-reversibility.

1 Laboratoire POEMS, ENSTA, 32 Boulevard Victor, 75739 Paris Cedex 15, France. laurent.bourgeois@ensta.fr 
of the boundary where limit conditions are unknown, and of logarithmic type in the whole domain, as it will be described again in the following paper. In the particular case of the Helmholtz equation, the authors of $[12,23]$ analyzed the influence of the frequency on the Hölder and the logarithmic stability estimates, precisely on the constant in front of these estimates.

Here we are interested in the influence of the regularity of the boundary on stability estimates, precisely on the optimal exponent of the logarithmic stability estimate in the whole domain. In [1], a logarithmic stability estimate is established in the case of a Lipschitz domain for functions of class $C^{1, \alpha}$ with $0<\alpha<1$, with the help of doubling inequalities. The exponent of the logarithm is however unspecified in that paper. In [6], a stability estimate is obtained in two dimensions in $C^{2, \alpha}$ class domains with $0<\alpha<1$ and for functions of class $C^{2}$. In [24], a stability estimate is obtained in domains of class $C^{2}$ for functions of class $H^{\eta}$, where $\eta>2$ depends on the dimension. In these two last papers, the exponent is specified but not proved to be optimal.

In the following paper, we specify the exponent of the logarithmic stability estimate in the case of a domain with $C^{1,1}$ boundary for functions in $H^{2}$. Precisely, we prove that this exponent is any $\kappa<1$ and that the value 1 cannot be improved. In this sense, our stability estimate in nearly optimal. The case of a domain with Lipschitz boundary, which requires a completely different technique, is considered in [3]. The choice of the functional space $H^{2}$ is motivated by a particular application of our stability estimate, which is the derivation of a convergence rate for the method of quasi-reversibility to regularize the ill-posed Cauchy problems for the elliptic operator $P[17]$. We hence obtain a nearly optimal convergence rate for the method of quasi-reversibility in the whole domain, which is new and completes the result of [16] concerning this convergence rate in a subdomain.

The starting point of our study is the nice article of Phung [21], who obtained the following conditional stability estimate for the operator $P=-\Delta .-k ., k \in \mathbb{R}$. For a bounded and connected domain $\Omega \subset \mathbb{R}^{N}$ of class $C^{\infty}$, if $\Gamma_{0}$ is an open part of $\partial \Omega$, then for all $\left.\kappa \in\right] 0,1\left[\right.$, there exist constants $C, \delta_{0}>0$ such that for all $\delta \in] 0, \delta_{0}\left[\right.$, for all function $u \in H^{2}(\Omega)$ which satisfies

$$
\|u\|_{H^{2}(\Omega)} \leq M, \quad\|P u\|_{L^{2}(\Omega)}+\|u\|_{H^{1}\left(\Gamma_{0}\right)}+\left\|\partial_{n} u\right\|_{L^{2}\left(\Gamma_{0}\right)} \leq \delta,
$$

where $M$ is a constant,

$$
\|u\|_{H^{1}(\Omega)} \leq C \frac{M}{(\log (M / \delta))^{\kappa}} .
$$

A similar estimate holds with $\|u\|_{H^{1}(\omega)}$ replacing $\|u\|_{H^{1}\left(\Gamma_{0}\right)}+\left\|\partial_{n} u\right\|_{L^{2}\left(\Gamma_{0}\right)}$ in (1.1) for any open domain $\omega \Subset \Omega$. The label "conditional" stems from the first inequality of (1.1), which is required to obtain stability. We also notice that despite $u \in H^{2}(\Omega)$, we only estimate $\|u\|_{H^{1}(\Omega)}$ in (1.2), which is due to the estimation of the function $u$ up to the part of the boundary $\partial \Omega$ which is complementary to $\Gamma_{0}$ (see the proof of Prop. 2.4). In [21], the proof of (1.2) for $C^{\infty}$ domains is mainly based on an interior Carleman estimate [8,11], as well as a Carleman estimate near the boundary [19]. Precisely, the analysis of stability near the boundary follows from a Carleman estimate in the half-space after using a local mapping from the Cartesian coordinates to the geodesic normal coordinates, which separates normal and tangential second derivatives in the principal part of the transformed operator. The Carleman estimates apply to the transformed operator and use microlocal analysis.

The aim of this paper is to prove that the stability estimate (1.2) still holds for domains of class $C^{1,1}$ with the same assumptions. Because it is based on geodesic normal coordinates, the technique used in [21] is not strictly speaking applicable to domains of class $C^{1,1}$. By definition of a $C^{1,1}$ domain, a particular mapping enables us to flatten the boundary and then probably to continue the analysis on the transformed operator in the spirit of [21], despite separation between normal and tangential second derivatives does not hold anymore.

The present paper is however devoted to an alternative technique that uses no local change of coordinates and which is based on the distance function to the boundary. Precisely, we use Carleman estimates near the boundary directly on the initial geometry and on the initial Laplace operator, by following the friendly method of [9] instead of microlocal analysis, and the exponential weight is a function of the distance to the boundary.

Our paper is organized as follows. The second section is devoted to the derivation of our stability estimate with the help of a Carleman inequality. This is based on the local regularity of the distance function to the boundary, 
which is related to the regularity of the domain. In Section 3 we prove that such stability estimate in nearly optimal. Lastly, in Section 4 we derive some convergence rates for the method of quasi-reversibility to regularize the ill-posed Cauchy problems.

\section{A stability estimate in Domains of Class $C^{1,1}$}

\subsection{About the regularity of the distance function}

We consider a bounded and connected domain $\Omega \subset \mathbb{R}^{N}$ of class $C^{1,1}$. For $x \in \bar{\Omega}$, we denote $d_{\partial \Omega}(x)$ the distance function to the boundary $\partial \Omega$, and we define the set

$$
\pi_{\partial \Omega}(x)=\left\{y \in \partial \Omega, \quad|x-y|=d_{\partial \Omega}(x)\right\},
$$

where |.| denotes the Euclidean norm in $\mathbb{R}^{N}$. At any point $y \in \partial \Omega$, the outward unit normal is denoted $n(y)$.

There are a number of contributions concerning the regularity of function $d_{\partial \Omega}$ near the boundary. Among these, the following theorem is proved in [7] (see Thm. 4.3, p. 219).

Theorem 2.1. If the domain $\Omega \subset \mathbb{R}^{N}$ is of class $C^{1,1}$, then for all $x_{0} \in \partial \Omega$, there exists a neighborhood $\mathcal{W}\left(x_{0}\right)$ of $x_{0}$ such that if $W\left(x_{0}\right)=\mathcal{W}\left(x_{0}\right) \cap \bar{\Omega}$,

$$
\forall x \in W\left(x_{0}\right), \quad \pi_{\partial \Omega}(x)=\left\{P_{\partial \Omega}(x)\right\}
$$

is a singleton and the map: $W\left(x_{0}\right) \rightarrow \mathbb{R}^{N}$

$$
x \mapsto P_{\partial \Omega}(x)
$$

is Lipschitz continuous in $W\left(x_{0}\right)$. Moreover,

$$
\forall x \in W\left(x_{0}\right), \quad \nabla d_{\partial \Omega}(x)=-n\left(P_{\partial \Omega}(x)\right) .
$$

As a result, $\nabla d_{\partial \Omega}$ is Lipschitz continuous in $W\left(x_{0}\right)$, so $d_{\partial \Omega} \in C^{1,1}\left(W\left(x_{0}\right)\right)$, in particular the components of $\nabla^{2} d_{\partial \Omega}$ belong to $L^{\infty}\left(W\left(x_{0}\right)\right)$.

Remark 2.1. As proved by a counterexample in [7], p. 222, when $\Omega$ is only of class $C^{1, \alpha}$, with $0 \leq \alpha<1$, then $d_{\partial \Omega}$ may be not differentiable in a neighborhood of $\partial \Omega$. In particular, $\nabla d_{\partial \Omega}$ is not a $C^{0}$ function in a neighborhood of $\partial \Omega$.

\subsection{A Carleman estimate near the boundary}

We consider $x_{0} \in \partial \Omega, R_{0}>0$, and the set $B=\Omega \cap B\left(x_{0}, R_{0}\right)$. We define $\tilde{H}_{0}^{2}(B)$ as the restrictions to $B$ of functions in $H_{0}^{2}\left(B\left(x_{0}, R_{0}\right)\right)$.

Let the function $\psi$ satisfy $\psi \in C^{1}(\bar{B}), \nabla \psi \neq 0$ on $\bar{B}$, and $\nabla^{2} \psi \in\left(L^{\infty}(B)\right)^{N \times N}$.

We define for $\varepsilon \geq 0$,

$$
K_{\varepsilon}=\{x \in \bar{B}, \quad \psi(x) \geq \varepsilon\} .
$$

In the following, $\psi$ is chosen such that only two cases occur (see Fig. 1). In the first case $K_{0}=\bar{B}$, the boundary of $K_{0}$ in then included in $\partial \Omega \cup \partial B\left(x_{0}, R_{0}\right)$ and we denote $\partial K_{0}=\bar{B} \cap \partial \Omega$. In the second case $\{x, \psi(x)>0\} \cap \partial \Omega=\emptyset$, the boundary of $K_{0}$ is then included in $\{x, \psi(x)=0\} \cup \partial B\left(x_{0}, R_{0}\right)$ and we denote $\partial K_{0}=\{x \in \bar{B}, \psi(x)=0\}$.

Denoting $\phi(x)=\mathrm{e}^{\alpha \psi(x)}$ for $\alpha>0$, we have the following lemma. 

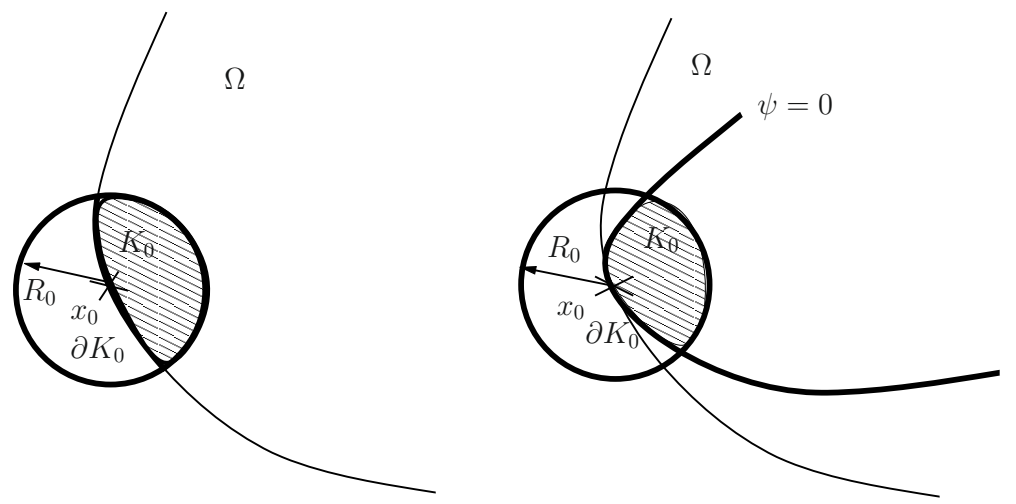

Figure 1. Two cases for definition of $K_{0}$ and $\partial K_{0}$.

Lemma 2.1. Let define $u \in C_{0}^{\infty}\left(B\left(x_{0}, R_{0}\right)\right)$ and $v=u \mathrm{e}^{\lambda \phi}$ with $\lambda>0$.

With the following definitions

$$
\begin{gathered}
p_{1}=2 \alpha^{2} \lambda \int_{K_{0}} \phi(\nabla \psi \cdot \nabla v)^{2} \mathrm{~d} x, \\
p_{2}=\alpha^{4} \lambda^{3} \int_{K_{0}} \phi^{3}|\nabla \psi|^{4} v^{2} \mathrm{~d} x, \quad p_{3}=\alpha^{2} \lambda \int_{K_{0}} \phi|\nabla \psi|^{2}|\nabla v|^{2} \mathrm{~d} x, \\
d_{1}=2 \alpha \lambda \int_{K_{0}} \phi \nabla^{t} v \cdot \nabla^{2} \psi \cdot \nabla v \mathrm{~d} x, \quad d_{2}=-\alpha \lambda \int_{K_{0}} \phi(\Delta \psi)|\nabla v|^{2} \mathrm{~d} x, \\
d_{3}=2 \alpha^{3} \lambda \int_{K_{0}} \phi|\nabla \psi|^{2}(\nabla \psi \cdot \nabla v) v \mathrm{~d} x, \quad d_{4}=4 \alpha^{2} \lambda \int_{K_{0}} \phi\left(\nabla^{t} \psi \cdot \nabla{ }^{2} \psi \cdot \nabla v\right) v \mathrm{~d} x, \\
d_{5}=2 \alpha^{3} \lambda^{3} \int_{K_{0}} \phi^{3}\left(\nabla^{t} \psi \cdot \nabla{ }^{2} \psi \cdot \nabla \psi\right) v^{2} \mathrm{~d} x, \quad d_{6}=\alpha^{3} \lambda^{3} \int_{K_{0}} \phi^{3}(\Delta \psi)|\nabla \psi|^{2} v^{2} \mathrm{~d} x, \\
b_{1}=-2 \alpha \lambda \int_{\partial K_{0}} \phi(\nabla \psi \cdot \nabla v) \frac{\partial v}{\partial n} \mathrm{~d} \Gamma, \quad b_{2}=\alpha \lambda \int_{\partial K_{0}} \phi \frac{\partial \psi}{\partial n}|\nabla v|^{2} \mathrm{~d} \Gamma, \\
p_{0}=\int_{\partial K_{0}} \phi|\nabla \psi|^{2} v \frac{\partial v}{\partial n} \mathrm{~d} \Gamma, \quad b_{4}=-\alpha^{3} \lambda^{3} \int_{\partial K_{0}} \phi^{3}|\nabla \psi|^{2} \frac{\partial \psi}{\partial n} v^{2} \mathrm{~d} \Gamma, \\
b_{3}=-2 \alpha^{2} \lambda \int_{K_{0}}(P u)^{2} \mathrm{e}^{2 \lambda \phi} \mathrm{d} x,
\end{gathered}
$$

we have

$$
p_{1}+p_{2}+p_{3}+d_{1}+d_{2}+d_{3}+d_{4}+d_{5}+d_{6}+b_{1}+b_{2}+b_{3}+b_{4} \leq p_{0}+p .
$$


Proof. We first find an expression of $P u$ as a function of $v$. Since $u=v \mathrm{e}^{-\lambda \phi}$,

$$
\begin{aligned}
\frac{\partial u}{\partial x_{j}}= & \left(\frac{\partial v}{\partial x_{j}}-\alpha \lambda \phi \frac{\partial \psi}{\partial x_{j}} v\right) \mathrm{e}^{-\lambda \phi} \\
\frac{\partial^{2} u}{\partial x_{j}^{2}}= & \left(\frac{\partial^{2} v}{\partial x_{j}^{2}}-\alpha \lambda \phi \frac{\partial^{2} \psi}{\partial x_{j}^{2}} v-\alpha^{2} \lambda \phi\left(\frac{\partial \psi}{\partial x_{j}}\right)^{2} v-\alpha \lambda \phi \frac{\partial \psi}{\partial x_{j}} \frac{\partial v}{\partial x_{j}}\right) \mathrm{e}^{-\lambda \phi} \\
& -\left(\frac{\partial v}{\partial x_{j}}-\alpha \lambda \phi \frac{\partial \psi}{\partial x_{j}} v\right) \alpha \lambda \phi \frac{\partial \psi}{\partial x_{j}} \mathrm{e}^{-\lambda \phi} \\
= & \left(\frac{\partial^{2} v}{\partial x_{j}{ }^{2}}-\alpha \lambda \phi \frac{\partial^{2} \psi}{\partial x_{j}^{2}} v-\alpha^{2} \lambda \phi\left(\frac{\partial \psi}{\partial x_{j}}\right)^{2} v-2 \alpha \lambda \phi \frac{\partial \psi}{\partial x_{j}} \frac{\partial v}{\partial x_{j}}+\alpha^{2} \lambda^{2} \phi^{2}\left(\frac{\partial \psi}{\partial x_{j}}\right)^{2} v\right) \mathrm{e}^{-\lambda \phi}
\end{aligned}
$$

whence

$$
\Delta u+k u=\left(\Delta v+k v-\alpha \lambda \phi(\Delta \psi) v-\alpha^{2} \lambda \phi|\nabla \psi|^{2} v-2 \alpha \lambda \phi(\nabla \psi \cdot \nabla v)+\alpha^{2} \lambda^{2} \phi^{2}|\nabla \psi|^{2} v\right) \mathrm{e}^{-\lambda \phi} .
$$

The above equation can be rewritten

$$
-P u \mathrm{e}^{\lambda \phi}=M_{1} v+M_{2} v+M_{3} v
$$

by denoting

$$
\begin{aligned}
& M_{1} v=\Delta v+\alpha^{2} \lambda^{2} \phi^{2}|\nabla \psi|^{2} v \\
& M_{2} v=-2 \alpha \lambda \phi(\nabla \psi \cdot \nabla v)-2 \alpha^{2} \lambda \phi|\nabla \psi|^{2} v \\
& M_{3} v=k v+\alpha^{2} \lambda \phi|\nabla \psi|^{2} v-\alpha \lambda \phi(\Delta \psi) v .
\end{aligned}
$$

It follows that

$$
\left\|M_{1} v+M_{2} v\right\|_{L^{2}\left(K_{0}\right)}^{2}=\left\|P u \mathrm{e}^{\lambda \phi}+M_{3} v\right\|_{L^{2}\left(K_{0}\right)}^{2}
$$

whence

$$
\left(M_{1} v, M_{2} v\right)_{L^{2}\left(K_{0}\right)} \leq\left\|P u \mathrm{e}^{\lambda \phi}\right\|_{L^{2}\left(K_{0}\right)}^{2}+\left\|M_{3} v\right\|_{L^{2}\left(K_{0}\right)}^{2}
$$

We now develop that left-hand side term. Since $M_{1} v$ and $M_{2} v$ are both the sum of two terms, with obvious notations we have

$$
\left(M_{1} v, M_{2} v\right)_{L^{2}\left(K_{0}\right)}=I_{11}+I_{12}+I_{21}+I_{22}
$$


By integration by parts in $K_{0}$, we obtain by using the Einstein notation for repeated indices,

$$
\begin{aligned}
I_{11}= & -2 \alpha \lambda \int_{K_{0}} \phi(\Delta v)(\nabla \psi \cdot \nabla v) \mathrm{d} x \\
= & 2 \alpha \lambda \int_{K_{0}} \frac{\partial v}{\partial x_{i}} \frac{\partial \cdot}{\partial x_{i}}\left(\phi \frac{\partial \psi}{\partial x_{j}} \frac{\partial v}{\partial x_{j}}\right) \mathrm{d} x-2 \alpha \lambda \int_{\partial K_{0}} \phi \frac{\partial v}{\partial n}(\nabla \psi \cdot \nabla v) \mathrm{d} \Gamma \\
= & 2 \alpha \lambda \int_{K_{0}} \phi \frac{\partial v}{\partial x_{i}} \frac{\partial^{2} \psi}{\partial x_{i} \partial x_{j}} \frac{\partial v}{\partial x_{j}} \mathrm{~d} x+2 \alpha^{2} \lambda \int_{K_{0}} \phi \frac{\partial v}{\partial x_{i}} \frac{\partial \psi}{\partial x_{i}} \frac{\partial \psi}{\partial x_{j}} \frac{\partial v}{\partial x_{j}} \mathrm{~d} x \\
& +2 \alpha \lambda \int_{K_{0}} \phi \frac{\partial v}{\partial x_{i}} \frac{\partial \psi}{\partial x_{j}} \frac{\partial^{2} v}{\partial x_{i} \partial x_{j}} \mathrm{~d} x-2 \alpha \lambda \int_{\partial K_{0}} \phi \frac{\partial v}{\partial n}(\nabla \psi \cdot \nabla v) \mathrm{d} \Gamma \\
= & 2 \alpha^{2} \lambda \int_{K_{0}} \phi(\nabla \psi \cdot \nabla v)^{2} \mathrm{~d} x+2 \alpha \lambda \int_{K_{0}} \phi \nabla^{t} v \cdot \nabla^{2} \psi \cdot \nabla v \mathrm{~d} x \\
& +\alpha \lambda \int_{K_{0}} \phi \nabla \psi \cdot \nabla\left(|\nabla v|^{2}\right) \mathrm{d} x-2 \alpha \lambda \int_{\partial K_{0}} \phi \frac{\partial v}{\partial n}(\nabla \psi \cdot \nabla v) \mathrm{d} \Gamma .
\end{aligned}
$$

The third term of the above sum can be rewritten

$$
\begin{aligned}
I_{11}^{\prime} & :=\alpha \lambda \int_{K_{0}} \phi \nabla \psi \cdot \nabla\left(|\nabla v|^{2}\right) \mathrm{d} x \\
& =-\alpha \lambda \int_{K_{0}} \frac{\partial \cdot}{\partial x_{i}}\left(\phi \frac{\partial \psi}{\partial x_{i}}\right)|\nabla v|^{2} \mathrm{~d} x+\alpha \lambda \int_{\partial K_{0}} \phi \frac{\partial \psi}{\partial n}|\nabla v|^{2} \mathrm{~d} \Gamma \\
& =-\alpha \lambda \int_{K_{0}} \phi(\Delta \psi)|\nabla v|^{2} \mathrm{~d} x-\alpha^{2} \lambda \int_{K_{0}} \phi|\nabla \psi|^{2}|\nabla v|^{2} \mathrm{~d} x+\alpha \lambda \int_{\partial K_{0}} \phi \frac{\partial \psi}{\partial n}|\nabla v|^{2} \mathrm{~d} \Gamma .
\end{aligned}
$$

Similarly, we have

$$
\begin{aligned}
& I_{12}=-2 \alpha^{2} \lambda \int_{K_{0}} \phi|\nabla \psi|^{2} v \Delta v \mathrm{~d} x \\
& =2 \alpha^{2} \lambda \int_{K_{0}} \nabla\left(\phi|\nabla \psi|^{2} v\right) \cdot \nabla v \mathrm{~d} x-2 \alpha^{2} \lambda \int_{\partial K_{0}} \phi|\nabla \psi|^{2} v \frac{\partial v}{\partial n} \mathrm{~d} \Gamma \\
& =2 \alpha^{3} \lambda \int_{K_{0}} \phi|\nabla \psi|^{2}(\nabla \psi \cdot \nabla v) v \mathrm{~d} x+2 \alpha^{2} \lambda \int_{K_{0}} \phi v \nabla\left(|\nabla \psi|^{2}\right) . \nabla v \mathrm{~d} x \\
& +2 \alpha^{2} \lambda \int_{K_{0}} \phi|\nabla \psi|^{2}|\nabla v|^{2} \mathrm{~d} x-2 \alpha^{2} \lambda \int_{\partial K_{0}} \phi|\nabla \psi|^{2} v \frac{\partial v}{\partial n} \mathrm{~d} \Gamma . \\
& I_{21}=-2 \alpha^{3} \lambda^{3} \int_{K_{0}} \phi^{3}|\nabla \psi|^{2}(\nabla \psi \cdot \nabla v) v \mathrm{~d} x=-\alpha^{3} \lambda^{3} \int_{K_{0}} \phi^{3}|\nabla \psi|^{2} \frac{\partial \psi}{\partial x_{i}} \frac{\partial\left(v^{2}\right)}{\partial x_{i}} \mathrm{~d} x \\
& =\alpha^{3} \lambda^{3} \int_{K_{0}} \operatorname{div}\left(\phi^{3}|\nabla \psi|^{2} \nabla \psi\right) v^{2} \mathrm{~d} x-\alpha^{3} \lambda^{3} \int_{\partial K_{0}} \phi^{3}|\nabla \psi|^{2} \frac{\partial \psi}{\partial n} v^{2} \mathrm{~d} \Gamma \\
& =3 \alpha^{4} \lambda^{3} \int_{K_{0}} \phi^{3}|\nabla \psi|^{4} v^{2} \mathrm{~d} x+\alpha^{3} \lambda^{3} \int_{K_{0}} \phi^{3} \operatorname{div}\left(|\nabla \psi|^{2} \nabla \psi\right) v^{2} \mathrm{~d} x \\
& -\alpha^{3} \lambda^{3} \int_{\partial K_{0}} \phi^{3}|\nabla \psi|^{2} \frac{\partial \psi}{\partial n} v^{2} \mathrm{~d} \Gamma \text {. }
\end{aligned}
$$


Lastly

$$
I_{22}=-2 \alpha^{4} \lambda^{3} \int_{K_{0}} \phi^{3}|\nabla \psi|^{4} v^{2} \mathrm{~d} x .
$$

If we add all terms and simplify, we finally obtain

$$
\left(M_{1} v, M_{2} v\right)_{L^{2}\left(K_{0}\right)}=p_{1}+p_{2}+p_{3}+d_{1}+d_{2}+d_{3}+d_{4}+d_{5}+d_{6}+b_{1}+b_{2}+b_{3}+b_{4} .
$$

Since

this completes the proof of the lemma.

$$
\left\|M_{3} v\right\|_{L^{2}\left(K_{0}\right)}^{2}=p_{0}, \quad\left\|P u \mathrm{e}^{\lambda \phi}\right\|_{L^{2}\left(K_{0}\right)}^{2}=p,
$$

We obtain the following Carleman estimate in $K_{0}$.

Proposition 2.1. There exist $K, \alpha_{0}, \lambda_{0}>0$ such that $\forall \alpha \geq \alpha_{0}, \forall \lambda \geq \lambda_{0}, \forall u \in \tilde{H}_{0}^{2}(B)$,

$$
\begin{aligned}
& \alpha^{4} \lambda^{3} \int_{K_{0}} \phi^{3}|\nabla \psi|^{4} u^{2} \mathrm{e}^{2 \lambda \phi} \mathrm{d} x+\alpha^{2} \lambda \int_{K_{0}} \phi|\nabla \psi|^{2}|\nabla u|^{2} \mathrm{e}^{2 \lambda \phi} \mathrm{d} x \\
& \leq K \int_{K_{0}}|P u|^{2} \mathrm{e}^{2 \lambda \phi} \mathrm{d} x+K \alpha \lambda \int_{\partial K_{0}} \phi|\nabla \psi||\nabla u|^{2} \mathrm{e}^{2 \lambda \phi} \mathrm{d} \Gamma+K \alpha^{3} \lambda^{3} \int_{\partial K_{0}} \phi^{3}|\nabla \psi|^{3} u^{2} \mathrm{e}^{2 \lambda \phi} \mathrm{d} \Gamma
\end{aligned}
$$

Proof. For $u \in C_{0}^{\infty}\left(B\left(x_{0}, R_{0}\right)\right)$, we denote $v=u \mathrm{e}^{\lambda \phi}$ and use the notations of Lemma 2.1. Since $\nabla \psi \neq 0$ on $\bar{B}$, we have

$$
\begin{aligned}
& p_{2}+d_{5}+d_{6} \geq \alpha^{3} \lambda^{3} \int_{K_{0}} \phi^{3}|\nabla \psi|^{4}\left(\alpha+\frac{2 \mu_{-}(\psi)+\Delta \psi}{|\nabla \psi|^{2}}\right) v^{2} \mathrm{~d} x, \\
& p_{3}+d_{1}+d_{2} \geq \alpha \lambda \int_{K_{0}} \phi|\nabla \psi|^{2}\left(\alpha+\frac{2 \mu_{-}(\psi)-\Delta \psi}{|\nabla \psi|^{2}}\right)|\nabla v|^{2} \mathrm{~d} x,
\end{aligned}
$$

where $\mu_{-}(\psi)$ (resp. $\left.\mu_{+}(\psi)\right)$ is the smallest (resp. largest) eigenvalue of $\nabla^{2} \psi$. Since $\mu_{-}(\psi)$ and $\Delta \psi$ belong to $L^{\infty}(B)$, there exists a constant $c$ such that

$$
\frac{2 \mu_{-}(\psi) \pm \Delta \psi}{|\nabla \psi|^{2}} \geq c \quad \text { a.e. in } K_{0} .
$$

Hence, for sufficiently large $\alpha$ there exist constants $K, K^{\prime}>0$ such that

$$
\begin{gathered}
p_{2}+d_{5}+d_{6} \geq K \alpha^{4} \lambda^{3} \int_{K_{0}} \phi^{3}|\nabla \psi|^{4} v^{2} \mathrm{~d} x, \\
p_{3}+d_{1}+d_{2} \geq K^{\prime} \alpha^{2} \lambda \int_{K_{0}} \phi|\nabla \psi|^{2}|\nabla v|^{2} \mathrm{~d} x .
\end{gathered}
$$

Now we look at terms $d_{3}$ and $d_{4}$.

$$
\left|d_{3}\right| \leq 2 \alpha^{3} \lambda \int_{K_{0}} \phi|\nabla \psi|^{2}|\nabla \psi \cdot \nabla v||v| \mathrm{d} x .
$$

By using Young's formula,

$$
\begin{aligned}
\left|d_{3}\right| & \leq \alpha^{2} \lambda \int_{K_{0}} \phi(\nabla \psi \cdot \nabla v)^{2} \mathrm{~d} x+\alpha^{4} \lambda \int_{K_{0}} \phi|\nabla \psi|^{4} v^{2} \mathrm{~d} x \\
& \leq \alpha^{2} \lambda \int_{K_{0}} \phi(\nabla \psi \cdot \nabla v)^{2} \mathrm{~d} x+\alpha^{4} \lambda \int_{K_{0}} \phi^{3}|\nabla \psi|^{4} v^{2} \mathrm{~d} x
\end{aligned}
$$

since $\phi \geq 1$ in $K_{0}$. 
Hence we have

$$
\begin{aligned}
p_{1}+d_{3} & \geq \alpha^{2} \lambda \int_{K_{0}} \phi(\nabla \psi \cdot \nabla v)^{2} \mathrm{~d} x-\alpha^{4} \lambda \int_{K_{0}} \phi^{3}|\nabla \psi|^{4} v^{2} \mathrm{~d} x \\
& \geq-\alpha^{4} \lambda \int_{K_{0}} \phi^{3}|\nabla \psi|^{4} v^{2} \mathrm{~d} x . \\
\left|d_{4}\right| & \leq 4 \alpha^{2} \lambda \int_{K_{0}} \phi \mu(\psi)|\nabla \psi||\nabla v||v| \mathrm{d} x
\end{aligned}
$$

with $\mu(\psi)=\max \left(\left|\mu_{-}(\psi)\right|,\left|\mu_{+}(\psi)\right|\right)$, and by using again Young's formula,

$$
\left|d_{4}\right| \leq 2 \alpha^{3} \lambda^{2} \int_{K_{0}} \phi \mu(\psi)|\nabla \psi|^{2} v^{2} \mathrm{~d} x+2 \alpha \int_{K_{0}} \phi \mu(\psi)|\nabla v|^{2} \mathrm{~d} x .
$$

Since $\mu(\psi) \in L^{\infty}(B)$, there exists a constant $C$ such that

$$
\frac{\mu(\psi)}{|\nabla \psi|^{2}} \leq C \quad \text { a.e. in } K_{0}
$$

Then, since $\phi \geq 1$ in $K_{0}$,

$$
\left|d_{4}\right| \leq 2 C \alpha^{3} \lambda^{2} \int_{K_{0}} \phi^{3}|\nabla \psi|^{4} v^{2} \mathrm{~d} x+2 C \alpha \int_{K_{0}} \phi|\nabla \psi|^{2}|\nabla v|^{2} \mathrm{~d} x .
$$

We now consider the case of $p_{0}$.

We have

$$
p_{0}=\alpha^{4} \lambda^{2} \int_{K_{0}} \phi^{2}|\nabla \psi|^{4}\left(1+\frac{k}{\alpha^{2} \lambda \phi|\nabla \psi|^{2}}-\frac{1}{\alpha} \frac{\Delta \psi}{|\nabla \psi|^{2}}\right)^{2} v^{2} \mathrm{~d} x .
$$

For $\lambda \geq 1$ and sufficiently large $\alpha$, we obtain

$$
p_{0} \leq 2 \alpha^{4} \lambda^{2} \int_{K_{0}} \phi^{3}|\nabla \psi|^{4} v^{2} \mathrm{~d} x
$$

If we gather all the above estimates, we obtain

$$
\begin{aligned}
& p_{1}+p_{2}+p_{3}+d_{1}+d_{2}+d_{3}+d_{4}+d_{5}+d_{6}-p_{0} \\
& \quad \geq K_{0} \alpha^{4} \lambda^{3} \int_{K_{0}} \phi^{3}|\nabla \psi|^{4} v^{2} \mathrm{~d} x+K_{1} \alpha^{2} \lambda \int_{K_{0}} \phi|\nabla \psi|^{2}|\nabla v|^{2} \mathrm{~d} x,
\end{aligned}
$$

with

$$
K_{0}=K-\frac{1}{\lambda^{2}}-\frac{2 C}{\alpha \lambda}-\frac{2}{\lambda}, \quad K_{1}=K^{\prime}-\frac{2 C}{\alpha \lambda}
$$

As a result, when $\alpha$ and $\lambda$ are large enough, we have $K_{0}, K_{1}>0$. 
Now let us consider $\left|b_{i}\right|, i=1,2,3,4$. We have

$$
\begin{aligned}
\left|b_{1}+b_{2}\right| & \leq 3 \alpha \lambda \int_{\partial K_{0}} \phi|\nabla \psi||\nabla v|^{2} \mathrm{~d} \Gamma \\
\left|b_{3}\right| & \leq 2 \alpha^{2} \lambda \int_{\partial K_{0}} \phi|\nabla \psi|^{2}|\nabla v||v| \mathrm{d} \Gamma \\
& \leq \alpha \lambda \int_{\partial K_{0}} \phi|\nabla \psi||\nabla v|^{2} \mathrm{~d} \Gamma+\alpha^{3} \lambda \int_{\partial K_{0}} \phi|\nabla \psi|^{3} v^{2} \mathrm{~d} \Gamma \\
\left|b_{4}\right| & \leq \alpha^{3} \lambda^{3} \int_{\partial K_{0}} \phi^{3}|\nabla \psi|^{3} v^{2} \mathrm{~d} \Gamma .
\end{aligned}
$$

Since $\phi \geq 1$, for $\lambda \geq 1$ we have

$$
\left|b_{1}+b_{2}+b_{3}+b_{4}\right| \leq 4 \alpha \lambda \int_{\partial K_{0}} \phi|\nabla \psi||\nabla v|^{2} \mathrm{~d} \Gamma+2 \alpha^{3} \lambda^{3} \int_{\partial K_{0}} \phi^{3}|\nabla \psi|^{3} v^{2} \mathrm{~d} \Gamma
$$

Applying Lemma 2.1, we obtain that for sufficiently large $\alpha, \lambda$, there exists a constant $K>0$ such that

$$
\begin{aligned}
\alpha^{4} \lambda^{3} \int_{K_{0}} \phi^{3}|\nabla \psi|^{4} v^{2} \mathrm{~d} x+\alpha^{2} \lambda \int_{K_{0}} \phi|\nabla \psi|^{2}|\nabla v|^{2} \mathrm{~d} x \leq & K \int_{K_{0}}|P u|^{2} \mathrm{e}^{2 \lambda \phi} \mathrm{d} x \\
& +\left.K \alpha \lambda \int_{\partial K_{0}} \phi|\nabla \psi| \nabla v\right|^{2} \mathrm{~d} \Gamma+K \alpha^{3} \lambda^{3} \int_{\partial K_{0}} \phi^{3}|\nabla \psi|^{3} v^{2} \mathrm{~d} \Gamma .
\end{aligned}
$$

Now we replace $v$ in the above estimate by its expression as a function of $u$.

$$
v=u \mathrm{e}^{\lambda \phi}, \quad \nabla v=(\nabla u) \mathrm{e}^{\lambda \phi}+\alpha \lambda \phi u(\nabla \psi) \mathrm{e}^{\lambda \phi} .
$$

We obtain

$$
\begin{aligned}
& 2 \alpha^{4} \lambda^{3} \int_{K_{0}} \phi^{3}|\nabla \psi|^{4} u^{2} \mathrm{e}^{2 \lambda \phi} \mathrm{d} x+\alpha^{2} \lambda \int_{K_{0}} \phi|\nabla \psi|^{2}|\nabla u|^{2} \mathrm{e}^{2 \lambda \phi} \mathrm{d} x+2 \alpha^{3} \lambda^{2} \int_{K_{0}} \phi^{2}|\nabla \psi|^{2} u(\nabla \psi \cdot \nabla u) \mathrm{e}^{2 \lambda \phi} \mathrm{d} x \\
& \leq K \int_{K_{0}}|P u|^{2} \mathrm{e}^{2 \lambda \phi} \mathrm{d} x+K \alpha \lambda \int_{\partial K_{0}} \phi|\nabla \psi||\nabla u|^{2} \mathrm{e}^{2 \lambda \phi} \mathrm{d} \Gamma \\
&+2 K \alpha^{2} \lambda^{2} \int_{\partial K_{0}} \phi^{2}|\nabla \psi| u(\nabla \psi \cdot \nabla u) \mathrm{e}^{2 \lambda \phi} \mathrm{d} \Gamma+2 K \alpha^{3} \lambda^{3} \int_{\partial K_{0}} \phi^{3}|\nabla \psi|^{3} u^{2} \mathrm{e}^{2 \lambda \phi} \mathrm{d} \Gamma
\end{aligned}
$$

We now use the following Young's inequalities:

$$
\left.\left.\left|2 \alpha^{3} \lambda^{2} \int_{K_{0}} \phi^{2}\right| \nabla \psi\right|^{2} u(\nabla \psi \cdot \nabla u) \mathrm{e}^{2 \lambda \phi} \mathrm{d} x\left|\leq \frac{1}{r} \alpha^{4} \lambda^{3} \int_{K_{0}} \phi^{3}\right| \nabla \psi\right|^{4} u^{2} \mathrm{e}^{2 \lambda \phi} \mathrm{d} x+\alpha^{2} \lambda r \int_{K_{0}} \phi|\nabla \psi|^{2}|\nabla u|^{2} \mathrm{e}^{2 \lambda \phi} \mathrm{d} x
$$

with $r>0$, and

$$
\left|2 \alpha^{2} \lambda^{2} \int_{\partial K_{0}} \phi^{2}\right| \nabla \psi\left|u(\nabla \psi \cdot \nabla u) \mathrm{e}^{2 \lambda \phi} \mathrm{d} \Gamma\right| \leq \alpha \lambda \int_{\partial K_{0}} \phi|\nabla \psi||\nabla u|^{2} \mathrm{e}^{2 \lambda \phi} \mathrm{d} \Gamma+\alpha^{3} \lambda^{3} \int_{\partial K_{0}} \phi^{3}|\nabla \psi|^{3} u^{2} \mathrm{e}^{2 \lambda \phi} \mathrm{d} \Gamma
$$


As a conclusion, if we choose $1 / 2<r<1$, we obtain $K>0$ such that for $\alpha, \lambda$ large enough, and for all $u \in C_{0}^{\infty}\left(B\left(x_{0}, R_{0}\right)\right)$,

$$
\begin{aligned}
& \alpha^{4} \lambda^{3} \int_{K_{0}} \phi^{3}|\nabla \psi|^{4} u^{2} \mathrm{e}^{2 \lambda \phi} \mathrm{d} x+\alpha^{2} \lambda \int_{K_{0}} \phi|\nabla \psi|^{2}|\nabla u|^{2} \mathrm{e}^{2 \lambda \phi} \mathrm{d} x \\
& \leq K \int_{K_{0}}|P u|^{2} \mathrm{e}^{2 \lambda \phi} \mathrm{d} x+K \alpha \lambda \int_{\partial K_{0}} \phi|\nabla \psi||\nabla u|^{2} \mathrm{e}^{2 \lambda \phi} \mathrm{d} \Gamma+K \alpha^{3} \lambda^{3} \int_{\partial K_{0}} \phi^{3}|\nabla \psi|^{3} u^{2} \mathrm{e}^{2 \lambda \phi} \mathrm{d} \Gamma .
\end{aligned}
$$

By density, the above result remains true for $u \in \tilde{H}_{0}^{2}(B)$.

Remark 2.2. In the following, we can use a simpler statement as in Proposition 2.1. Once we have fixed $\alpha=\alpha_{0}>0$, we can drop $\alpha$ and $\psi$ from the Carleman inequality and obtain there exist $K, \lambda_{0}>0$ such that $\forall \lambda \geq \lambda_{0}, \forall u \in \tilde{H}_{0}^{2}(B)$,

$$
\lambda^{3} \int_{K_{0}} u^{2} \mathrm{e}^{2 \lambda \phi} \mathrm{d} x+\lambda \int_{K_{0}}|\nabla u|^{2} \mathrm{e}^{2 \lambda \phi} \mathrm{d} x \leq K \int_{K_{0}}|P u|^{2} \mathrm{e}^{2 \lambda \phi} \mathrm{d} x+K \lambda \int_{\partial K_{0}}|\nabla u|^{2} \mathrm{e}^{2 \lambda \phi} \mathrm{d} \Gamma+K \lambda^{3} \int_{\partial K_{0}} u^{2} \mathrm{e}^{2 \lambda \phi} \mathrm{d} \Gamma .
$$

We hence obtain the same Carleman inequality as in Proposition 2.1 from [19].

\subsection{Two stability estimates near the boundary}

We consider a bounded and connected domain $\Omega \subset \mathbb{R}^{N}$ with a $C^{1,1}$ boundary $\partial \Omega$, and $\Gamma_{0}$ an open domain of $\partial \Omega$. This implies that there exist $x_{0} \in \Gamma_{0}$ and $\tau>0$ with $\partial \Omega \cap B\left(x_{0}, \tau\right) \subset \Gamma_{0}$.

In this section we apply the Carleman estimate of Proposition 2.1 to obtain two stability estimates near the boundary. We use approximately the same method as in [21], with however two main differences. First, we use Carleman estimates involving weights $\mathrm{e}^{\alpha \psi_{1}}, \mathrm{e}^{\alpha \psi_{2}}$, where the functions $\psi_{1}, \psi_{2}$ are defined hereafter and depend on the distance function to the boundary, instead of a Carleman estimate in the half-space after a local change of coordinates. Second, as concerns Proposition 2.4, we use the level curves of a well-chosen weight instead of a perturbation of the domain in order to introduce the open domain $\omega_{1} \Subset \Omega$ in the right-hand side of the estimate. Before deriving these two stability estimates, we recall the following useful proposition, which is proved in [21] with the help of an interior Carleman estimate, and which is not influenced by the regularity of the domain.

Proposition 2.2. Let $\omega_{0}, \omega_{1}$ be two open domains such that $\omega_{0}, \omega_{1} \Subset \Omega$. There exist $s, c, \varepsilon_{0}>0$ such that $\forall \varepsilon \in] 0, \varepsilon_{0}\left[, \forall u \in H^{2}(\Omega)\right.$,

$$
\|u\|_{H^{1}\left(\omega_{1}\right)} \leq \frac{c}{\varepsilon}\left(\|P u\|_{L^{2}(\Omega)}+\|u\|_{H^{1}\left(\omega_{0}\right)}\right)+\varepsilon^{s}\|u\|_{H^{1}(\Omega)} .
$$

For all $x_{0} \in \partial \Omega$, we can choose the set $W\left(x_{0}\right)$ in Theorem 2.1 as $\bar{B}$ where $B=\Omega \cap B\left(x_{0}, R_{0}\right)$, for some $R_{0}$ with $0<R_{0}<1$. In the following, we will use the two functions $\psi_{1}, \psi_{2}$ defined in $\bar{\Omega}$ by:

$$
\begin{gathered}
\psi_{1}(x)=R-d_{\partial \Omega}(x)-\frac{1}{2} r(x)^{2}, \\
\psi_{2}(x)=\gamma \circ r(x) d_{\partial \Omega}(x)+(1-\gamma \circ r(x)) \tilde{d}_{\partial \Omega}(x), \\
\tilde{d}_{\partial \Omega}(x)=d_{\partial \Omega}(x)+\frac{1}{2}\left(d_{\partial \Omega}(x)^{2}-r(x)^{2}\right),
\end{gathered}
$$

with $r(x)=\left|x-x_{0}\right|$.

Here, $R>0$ is chosen such that $\psi_{1}>0$ on $\bar{B}$. We easily prove that for sufficiently small $R_{0}$ and $r_{0}<R_{0}$, $\{\tilde{d}(x)>\varepsilon\} \cap B\left(x_{0}, R_{0}\right) \neq \emptyset$ for all $\varepsilon$ with $0 \leq \varepsilon \leq r_{0}$. Furthermore, $\gamma$ is a $C^{2}$ function on $\left[0, R_{0}\right]$ such that $\gamma=1$ on the segment $\left[0, r_{0}\right]$, and which is non increasing on $\left[r_{0}, R_{0}\right]$ with $0<\gamma\left(R_{0}\right)<1$. Lastly we assume that $\gamma^{\prime}(r)+2 \gamma(r)>0$ on $\left[0, R_{0}\right]$. Such a function $\gamma$ exists, take for example $\gamma(r)=\tilde{\gamma}\left(r-r_{0}\right)$ for $r \in\left[r_{0}, R_{0}\right]$ with 
$\tilde{\gamma}(r)=\left(2 r^{2}+2 r+1\right) \mathrm{e}^{-2 r}$. Since $\gamma(r) \in[0,1]$, we have $\left\{\psi_{2}(x)>\varepsilon\right\} \cap B\left(x_{0}, R_{0}\right) \neq \emptyset$ for all $\varepsilon$ with $0 \leq \varepsilon \leq r_{0}$. We have the following result.

Lemma 2.2. The two functions $\psi_{1}$ and $\psi_{2}$ satisfy the following properties: for $i=1,2, \psi_{i} \in C^{1}(\bar{B}), \nabla \psi_{i} \neq 0$ on $\bar{B}$, and $\nabla^{2} \psi_{i} \in\left(L^{\infty}(B)\right)^{N \times N}$.

Proof. Theorem 2.1 implies that $d_{\partial \Omega}(x) \in C^{1}(\bar{B})$ and $\nabla^{2} d_{\partial \Omega} \in\left(L^{\infty}(B)\right)^{N \times N}$, which implies the same properties for $\psi_{1}$ and $\psi_{2}$.

We first verify that $\nabla \psi_{1} \neq 0$ in $\bar{B}$. Using Theorem 2.1 , we obtain that in $\bar{B}$,

$$
\nabla \psi_{1}(x)=n(y)-\left(x-x_{0}\right),
$$

where $y=P_{\partial \Omega}(x)$. If for some $x \in \bar{B}$ we had $\nabla \psi_{1}(x)=0$, then we would have $\left|x-x_{0}\right|=1$, which is impossible since $R_{0}<1$.

We consider now $\nabla \psi_{2}$. A straightforward calculation leads to

$$
\nabla \psi_{2}=\nabla d_{\partial \Omega}-\frac{1}{2} \nabla(\gamma \circ r)\left(d_{\partial \Omega}^{2}-r^{2}\right)+(1-\gamma \circ r)\left(d_{\partial \Omega} \nabla d_{\partial \Omega}-\left(x-x_{0}\right)\right) .
$$

Now using the fact that $\nabla d_{\partial \Omega}=-n(y)$ and $\nabla(\gamma \circ r)=\gamma^{\prime} \circ r(x)\left(x-x_{0}\right) /\left|x-x_{0}\right|$, we obtain

$$
\nabla \psi_{2}=-n(y)-\frac{1}{2} \gamma^{\prime}(r)\left(d_{\partial \Omega}^{2}-r^{2}\right) \frac{x-x_{0}}{\left|x-x_{0}\right|}+(1-\gamma(r))\left(-d_{\partial \Omega} n(y)-\left(x-x_{0}\right)\right) .
$$

If $x \in \bar{b}$ with $b=\Omega \cap B\left(0, r_{0}\right)$, then $\nabla \psi_{2}=-n(y) \neq 0$. Now assume that $\nabla \psi_{2}(x)=0$ for some $x \in \bar{B} \backslash \bar{b}$. For any $\tau(y) \perp n(y)$, we have

$$
\nabla \psi_{2}(x) \cdot \tau(y)=0=-\left(x-x_{0}\right) \cdot \tau\left(\frac{1}{2} \frac{\gamma^{\prime}(r)}{\left|x-x_{0}\right|}\left(d_{\partial \Omega}^{2}-r^{2}\right)+1-\gamma(r)\right) .
$$

Since $d_{\partial \Omega}(x) \leq r(x)$ on $\bar{B}, \gamma^{\prime} \leq 0$ and $1-\gamma>0$ on $\left.] r_{0}, R_{0}\right]$, we have necessarily $\left(x-x_{0}\right) \cdot \tau(y)=0$, whence $x-x_{0}=-\eta n(y)$ for some $\eta \in \mathbb{R}$.

Furthermore,

$$
\nabla \psi_{2}(x) . n(y)=0=-1+\frac{1}{2} \gamma^{\prime}(r)\left(d_{\partial \Omega}^{2}-\eta^{2}\right) \operatorname{sgn}(\eta)-(1-\gamma(r))\left(d_{\partial \Omega}-\eta\right),
$$

that is

$$
-\frac{1}{2} \gamma^{\prime}(r)\left(\eta^{2}-d_{\partial \Omega}^{2}\right) \operatorname{sgn}(\eta)+(1-\gamma(r))\left(\eta-d_{\partial \Omega}\right)=1 .
$$

But, since $\gamma^{\prime} \leq 0,1-\gamma>0$ and $d_{\partial \Omega} \leq|\eta| \leq R_{0}<1$,

$$
-\frac{1}{2} \gamma^{\prime}(r)\left(\eta^{2}-d_{\partial \Omega}^{2}\right) \operatorname{sgn}(\eta)+(1-\gamma(r))\left(\eta-d_{\partial \Omega}\right) \leq-\frac{1}{2} \gamma^{\prime}(r)+1-\gamma(r),
$$

and $-\gamma^{\prime} / 2+1-\gamma<1$ since $\gamma^{\prime}+2 \gamma>0$, which is a contradiction.

Now we prove the two following estimates.

Proposition 2.3. Let $x_{0} \in \Gamma_{0}$ and $\tau>0$ such that $\partial \Omega \cap B\left(x_{0}, \tau\right) \subset \Gamma_{0}$. There exists a neighborhood $\omega_{0}$ of $x_{0}$, there exist $s, c, \varepsilon_{0}>0$ such that $\left.\forall \varepsilon \in\right] 0, \varepsilon_{0}\left[, \forall u \in H^{2}(\Omega)\right.$,

$$
\|u\|_{H^{1}\left(\Omega \cap \omega_{0}\right)} \leq \frac{c}{\varepsilon}\left(\|P u\|_{L^{2}(\Omega)}+\|u\|_{H^{1}\left(\Gamma_{0}\right)}+\left\|\partial_{n} u\right\|_{L^{2}\left(\Gamma_{0}\right)}\right)+\varepsilon^{s}\|u\|_{H^{1}(\Omega)} .
$$


Proposition 2.4. Let $x_{0} \in \partial \Omega$. There exist a neighborhood $\omega$ of $x_{0}$ and an open domain $\omega_{1} \Subset \Omega$ such that for all $\kappa \in] 0,1\left[\right.$, there exist $c, \varepsilon_{0}>0$ such that $\left.\forall \varepsilon \in\right] 0, \varepsilon_{0}\left[, \forall u \in H^{2}(\Omega)\right.$,

$$
\|u\|_{H^{1}(\Omega \cap \omega)} \leq \mathrm{e}^{c / \varepsilon}\left(\|P u\|_{L^{2}(\Omega)}+\|u\|_{H^{1}\left(\omega_{1}\right)}\right)+\varepsilon^{\kappa}\|u\|_{H^{2}(\Omega)} .
$$

Proof of Proposition 2.3. We apply Proposition 2.1 and Remark 2.2 with function $\psi=\psi_{1}$ defined by (2.1). Here $K_{0}=\bar{B}$ since $\psi_{1}>0$ on $\bar{B}$ and $\partial K_{0}=\bar{B} \cap \partial \Omega$ (see the definition at the beginning of Sect. 2.2 and the left-hand side of Fig. 1). We assume that $R_{0}<\tau$ so that $\partial K_{0} \subset \Gamma_{0}$. We consider $z_{0}$ and $z_{1}$ such that $0<z_{1}<z_{0}<R$, with $\sqrt{2\left(R-z_{1}\right)}<R_{0}$. This last condition implies that $\left\{x \in \bar{\Omega}, \psi_{1}(x) \geq z_{1}\right\} \subset B\left(x_{0}, R_{0}\right)$. Next, we define $v=\chi u$, where $\chi$ is a function in $C_{0}^{\infty}\left(B\left(x_{0}, R_{0}\right)\right)$ such that $\chi=1$ on $K_{z_{1}}$.

Thus we have $v \in \tilde{H}_{0}^{2}(B)$, and there exist $K, \lambda_{0}>0$ such that for fixed (sufficiently large) $\alpha$ and for all $\lambda \geq \lambda_{0}$,

$$
\int_{K_{0}}\left(v^{2}+|\nabla v|^{2}\right) \mathrm{e}^{2 \lambda \phi} \mathrm{d} x \leq K \int_{K_{0}}|P v|^{2} \mathrm{e}^{2 \lambda \phi} \mathrm{d} x+K \lambda^{2} \int_{\partial K_{0}}\left(v^{2}+|\nabla v|^{2}\right) \mathrm{e}^{2 \lambda \phi} \mathrm{d} \Gamma .
$$

We hence obtain

$$
\begin{aligned}
\int_{K_{z_{0}}}\left(u^{2}+|\nabla u|^{2}\right) \mathrm{e}^{2 \lambda \phi} \mathrm{d} x \leq K^{\prime} \int_{K_{0}}|P u|^{2} \mathrm{e}^{2 \lambda \phi} \mathrm{d} x & \\
& +K^{\prime} \int_{K_{0} \backslash K_{z_{1}}}\left(u^{2}+|\nabla u|^{2}\right) \mathrm{e}^{2 \lambda \phi} \mathrm{d} x+K^{\prime} \lambda^{2} \int_{\partial K_{0}}\left(u^{2}+|\nabla u|^{2}\right) \mathrm{e}^{2 \lambda \phi} \mathrm{d} \Gamma .
\end{aligned}
$$

By denoting $h(z)=\mathrm{e}^{\alpha z}$, and since $\psi_{1} \geq z_{0}$ in $K_{z_{0}}, \psi_{1} \leq R$ in $K_{0}$ and $\psi_{1}<z_{1}$ in $K_{0} \backslash K_{z_{1}}$ (see the left-hand side of Fig. 2), it follows that

$$
\begin{aligned}
\mathrm{e}^{2 \lambda h\left(z_{0}\right)}\|u\|_{H^{1}\left(K_{z_{0}}\right)}^{2} \leq & K^{\prime} \mathrm{e}^{2 \lambda h(R)}\|P u\|_{L^{2}\left(K_{0}\right)}^{2}+K^{\prime} \mathrm{e}^{2 \lambda h\left(z_{1}\right)}\|u\|_{H^{1}\left(K_{0}\right)}^{2} \\
& +K^{\prime} \lambda^{2} \mathrm{e}^{2 \lambda h(R)}\left(\|u\|_{H^{1}\left(\partial K_{0}\right)}^{2}+\left\|\partial_{n} u\right\|_{L^{2}\left(\partial K_{0}\right)}^{2}\right)
\end{aligned}
$$

and thus for sufficiently large $\lambda$,

$$
\begin{aligned}
\|u\|_{H^{1}\left(K_{z_{0}}\right) \leq} & K^{\prime \prime} \lambda \mathrm{e}^{\lambda\left(h(R)-h\left(z_{0}\right)\right)}\left(\|P u\|_{L^{2}\left(K_{0}\right)}+\|u\|_{H^{1}\left(\partial K_{0}\right)}+\left\|\partial_{n} u\right\|_{L^{2}\left(\partial K_{0}\right)}\right) \\
& +K^{\prime \prime} \mathrm{e}^{-\lambda\left(h\left(z_{0}\right)-h\left(z_{1}\right)\right)}\|u\|_{H^{1}\left(K_{0}\right)} .
\end{aligned}
$$

Taking into account the fact that $h(R)-h\left(z_{0}\right)>0$ and $h\left(z_{0}\right)-h\left(z_{1}\right)>0$, by changing variable $\lambda \rightarrow \varepsilon$ we obtain that there exist $s, c, \varepsilon_{0}>0$ such that for all $\varepsilon, 0<\varepsilon<\varepsilon_{0}$, for all $u \in H^{2}(\Omega)$,

$$
\|u\|_{H^{1}\left(K_{z_{0}}\right)} \leq \frac{c}{\varepsilon}\left(\|P u\|_{L^{2}\left(K_{0}\right)}+\|u\|_{H^{1}\left(\partial K_{0}\right)}+\left\|\partial_{n} u\right\|_{L^{2}\left(\partial K_{0}\right)}\right)+\varepsilon^{s}\|u\|_{H^{1}\left(K_{0}\right)} .
$$

This ends the proof since $K_{0} \subset \bar{\Omega}, \partial K_{0} \subset \Gamma_{0}$ and $K_{z_{0}}=\left\{x \in \bar{\Omega}, d_{\partial \Omega}(x)+r^{2}(x) / 2 \leq R-z_{0}\right\}$ can be written $\overline{\Omega \cap \omega_{0}}$, where $\omega_{0}$ is a neighborhood of $x_{0}$.

In order to prove Proposition 2.4, we need the two following lemmas.

Lemma 2.3. Let $s, \beta, A$ and $B$ denote four non negative reals such that $\beta \leq B$. If $\exists c, \varepsilon_{0}>0$ such that $\forall \varepsilon$, $0<\varepsilon<\varepsilon_{0}$,

then

$$
\beta \leq \frac{c}{\varepsilon} A+\varepsilon^{s} B
$$

$$
\beta \leq C A^{\frac{s}{s+1}} B^{\frac{1}{s+1}}
$$



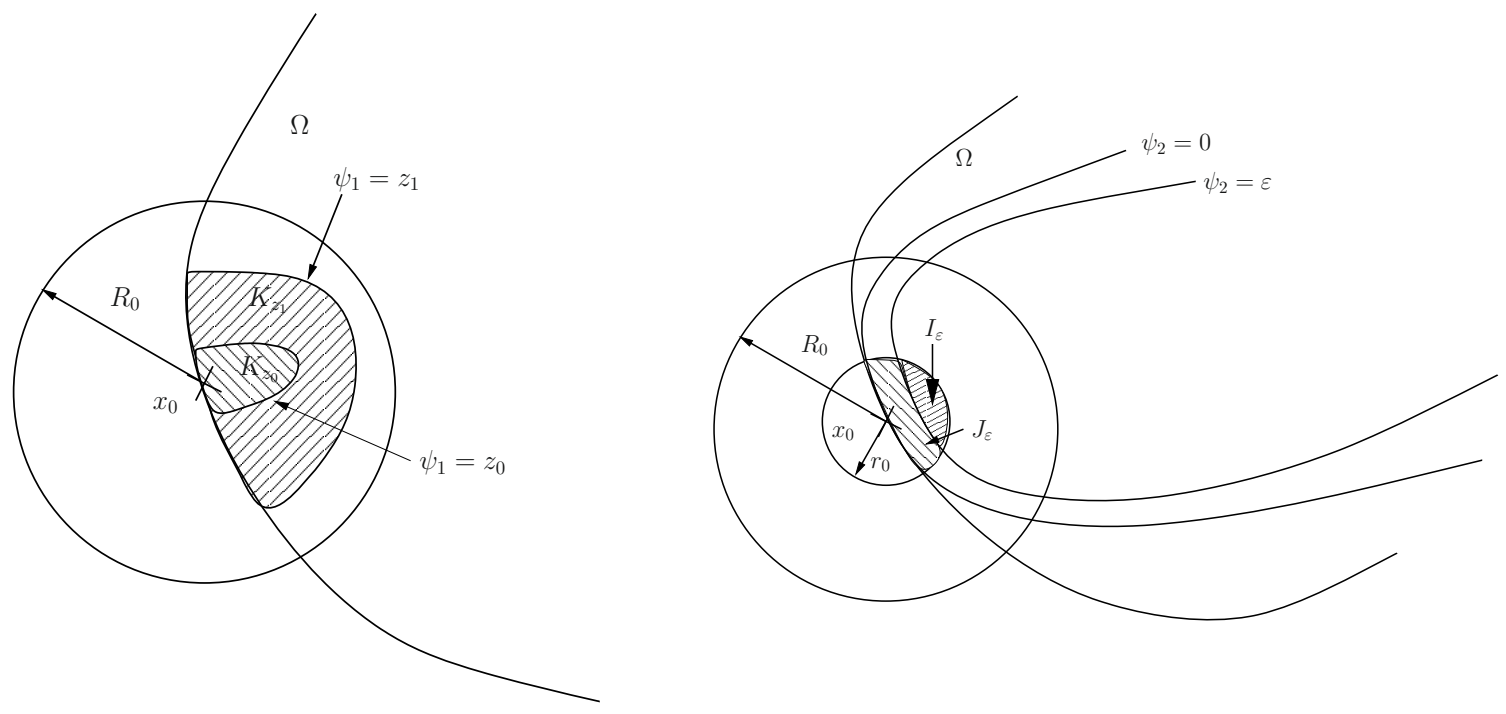

Figure 2. Left: proof of Proposition 2.3. Right: proof of Proposition 2.4.

where $C(s)=\max (D(s), \tilde{D}(s))$,

$$
D(s)=c^{\frac{s}{s+1}}\left(s^{\frac{1}{s+1}}+s^{-\frac{s}{s+1}}\right), \quad \tilde{D}(s)=\left(c / s \varepsilon_{0}^{(s+1)}\right)^{\frac{s}{s+1}} .
$$

$C(s)$ is a bounded function on each interval $\left[0, s_{0}\right]$.

Proof. We denote $\varepsilon_{\min }$ and $f_{\min }$ the minimizer and the minimum of

$$
f(\varepsilon)=\frac{c}{\varepsilon} A+\varepsilon^{s} B
$$

respectively, that is

$$
\varepsilon_{\min }=\left(\frac{c A}{s B}\right)^{\frac{1}{s+1}}, \quad f_{\min }=D(s) A^{\frac{s}{s+1}} B^{\frac{1}{s+1}}
$$

with

$$
D(s)=c^{\frac{s}{s+1}}\left(s^{\frac{1}{s+1}}+s^{-\frac{s}{s+1}}\right) .
$$

One should distinguish two cases. First, if $\varepsilon_{0}>\varepsilon_{\text {min }}$, the result follows with $C=D(s)$.

If $\varepsilon_{0} \leq \varepsilon_{\min }$, one has

$$
\varepsilon_{0} \leq\left(\frac{c A}{s B}\right)^{\frac{1}{s+1}}
$$

and hence

Using assumption $\beta \leq B$, we obtain

$$
B \leq A\left(c / s \varepsilon_{0}^{(s+1)}\right)
$$

$$
\beta \leq B^{\frac{s}{s+1}} B^{\frac{1}{s+1}} \leq \tilde{D}(s) A^{\frac{s}{s+1}} B^{\frac{1}{s+1}}
$$

with

$$
\tilde{D}(s)=\left(c / s \varepsilon_{0}^{(s+1)}\right)^{\frac{s}{s+1}},
$$


and the result follows with $C=\tilde{D}(s)$. To prove that $C(s)$ is a bounded function of $s \in\left[0, s_{0}\right]$ for fixed $\varepsilon_{0}$, we just have to verify that $D(s)$ and $\tilde{D}(s)$ are continuous on $\left[0, s_{0}\right]$, in particular at 0 .

Lemma 2.4. If $\Omega \subset \mathbb{R}^{N}$ is a bounded, connected and Lipschitz continuous domain, and if $d_{\partial \Omega}(x)$ denotes the distance of $x$ to $\partial \Omega$, then $\forall r \in] 0,1 / 2\left[, \forall u \in H^{r}(\Omega)\right.$,

$$
\left\|\frac{u}{d_{\partial \Omega}^{r}}\right\|_{L^{2}(\Omega)} \leq C\|u\|_{H^{r}(\Omega)}
$$

with $C>0$ depending only on $r$ and on $\Omega$.

Lemma 2.4 is known as Hardy's inequality and is proved for example in [10], p. 6.

Proof of Proposition 2.4. The first step consists in finding an estimate far away from $x_{0}$, by applying Proposition 2.1 and Remark 2.2 with function $\psi=\psi_{2}$ defined by (2.2)-(2.3). Here $K_{0}=\left\{x \in \bar{B}, \psi_{2}(x) \geq 0\right\}$ and $\partial K_{0}=\left\{x \in \bar{B}, \psi_{2}(x)=0\right\}$ (see the definition at the beginning of Sect. 2.2 and the right-hand side of Fig. 1). We consider the domains $K_{z, z^{\prime}}=\left\{x \in \bar{B}, z \leq \psi_{2}(x) \leq z^{\prime}\right\}$, with $0 \leq z<z^{\prime} \leq r_{0}$. For $v \in \tilde{H}_{0}^{2}(B)$, there exist $K, \lambda_{0}>0$ such that for fixed (sufficiently large) $\alpha \geq 1$ and for all $\lambda \geq \lambda_{0}$,

$$
\int_{K_{0}}\left(v^{2}+|\nabla v|^{2}\right) \mathrm{e}^{2 \lambda \phi} \mathrm{d} x \leq K \int_{K_{0}}|P v|^{2} \mathrm{e}^{2 \lambda \phi} \mathrm{d} x+K \lambda^{2} \int_{\partial K_{0}}\left(v^{2}+|\nabla v|^{2}\right) \mathrm{e}^{2 \lambda \phi} \mathrm{d} \Gamma .
$$

Let $\varepsilon$ be such that $0<\varepsilon<r_{0}$. Denoting again $h(z)=\mathrm{e}^{\alpha z}$, since $\psi_{2} \geq \varepsilon$ in $K_{\varepsilon, r_{0}}, \psi_{2} \leq R_{0}$ in $K_{0}$ and $\psi_{2}=0$ on $\partial K_{0}$, we obtain

$$
\mathrm{e}^{2 \lambda h(\varepsilon)}\|v\|_{H^{1}\left(K_{\varepsilon, r_{0}}\right)}^{2} \leq K \mathrm{e}^{2 \lambda h\left(R_{0}\right)}\|P v\|_{L^{2}\left(K_{0}\right)}^{2}+K \lambda^{2} \mathrm{e}^{2 \lambda h(0)}\left(\|v\|_{H^{1}\left(\partial K_{0}\right)}^{2}+\left\|\partial_{n} v\right\|_{L^{2}\left(\partial K_{0}\right)}^{2}\right),
$$

and hence, by using a classical trace theorem,

$$
\|v\|_{H^{1}\left(K_{\varepsilon, r_{0}}\right)} \leq K^{\prime} \mathrm{e}^{\lambda\left(h\left(R_{0}\right)-h(\varepsilon)\right)}\|P v\|_{L^{2}\left(K_{0}\right)}+K^{\prime} \lambda \mathrm{e}^{-\lambda(h(\varepsilon)-h(0))}\|v\|_{H^{2}\left(K_{0}\right)} .
$$

We notice that $h(\varepsilon)-h(0) \geq \alpha \varepsilon \geq \varepsilon$ and $\lambda \leq(2 / \varepsilon) \mathrm{e}^{\varepsilon \lambda / 2}$, whence there exist $d, L>0$ such that

$$
\|v\|_{H^{1}\left(K_{\varepsilon, r_{0}}\right)} \leq L \mathrm{e}^{d \lambda}\|P v\|_{L^{2}\left(K_{0}\right)}+L \frac{1}{\varepsilon} \mathrm{e}^{-\varepsilon \lambda}\|v\|_{H^{2}\left(K_{0}\right)} .
$$

Next, $s>0$ and $\mu>0$ are uniquely defined by $\mathrm{e}^{d \lambda}=1 / \mu$ and $\mathrm{e}^{-\varepsilon \lambda}=\mu^{s}$. It follows in particular that $s=\varepsilon / d$, and for $0<\mu \leq \mu_{0}=\mathrm{e}^{-d \lambda_{0}}, \forall v \in \tilde{H}_{0}^{2}(B)$,

$$
\varepsilon\|v\|_{H^{1}\left(K_{\varepsilon, r_{0}}\right)} \leq \frac{1}{\mu} L \varepsilon\|P v\|_{L^{2}\left(K_{0}\right)}+\mu^{s} L\|v\|_{H^{2}\left(K_{0}\right)} .
$$

We apply Lemma 2.3 with $s=\varepsilon / d, \beta=\varepsilon\|v\|_{H^{1}\left(K_{\varepsilon, r_{0}}\right)}, A=L \varepsilon\|P v\|_{L^{2}\left(K_{0}\right)}$ and $B=L\|v\|_{H^{2}\left(K_{0}\right)}$. There exists $C$ (independent of $\varepsilon$ ) such that for $\varepsilon$ with $0<\varepsilon<r_{0}$, for $v \in \tilde{H}_{0}^{2}(B)$,

$$
\|v\|_{H^{1}\left(K_{\varepsilon, r_{0}}\right)} \leq C\left(\|P v\|_{L^{2}\left(K_{0}\right)}\right)^{\frac{\varepsilon}{\varepsilon+d}}\left(\frac{1}{\varepsilon}\|v\|_{H^{2}\left(K_{0}\right)}\right)^{\frac{d}{\varepsilon+d}} .
$$

At this step we reproduce exactly the same calculations as in [21]. We introduce now $s>0$, such that

$$
\left(\varepsilon^{-\frac{d}{\varepsilon}(s+1)}\right)^{\frac{\varepsilon}{\varepsilon+d}}\left(\varepsilon^{s}\right)^{\frac{d}{\varepsilon+d}}=\left(\frac{1}{\varepsilon}\right)^{\frac{d}{\varepsilon+d}}
$$


it follows that

$$
\|v\|_{H^{1}\left(K_{\varepsilon, r_{0}}\right)} \leq C\left(\varepsilon^{-\frac{d}{\varepsilon}(s+1)}\|P v\|_{L^{2}\left(K_{0}\right)}\right)^{\frac{\varepsilon}{\varepsilon+d}}\left(\varepsilon^{s}\|v\|_{H^{2}\left(K_{0}\right)}\right)^{\frac{d}{\varepsilon+d}}
$$

Moreover,

$$
\varepsilon^{-\frac{d}{\varepsilon}(s+1)}=\mathrm{e}^{\frac{d}{\varepsilon}(s+1) \log \frac{1}{\varepsilon}}
$$

and for small $\varepsilon$, if we introduce $\mu>1$,

$$
\frac{1}{\varepsilon} \log \frac{1}{\varepsilon} \leq \frac{1}{\mu-1} \frac{1}{\varepsilon^{\mu}}
$$

(which is obtained by remarking that $\log 1 / \varepsilon^{\mu-1} \leq 1 / \varepsilon^{\mu-1}$ for small $\varepsilon$ ). This leads to

$$
\varepsilon^{-\frac{d}{\varepsilon}(s+1)} \leq \mathrm{e}^{\frac{d(s+1)}{(\mu-1) \varepsilon^{\mu}}}
$$

and finally, $\forall s>0, \forall \mu>1, \exists c>0$ such that for sufficiently small $\varepsilon, \forall v \in \tilde{H}_{0}^{2}(B)$,

$$
\|v\|_{H^{1}\left(K_{\varepsilon, r_{0}}\right)} \leq C\left(\mathrm{e}^{c / \varepsilon^{\mu}}\|P v\|_{L^{2}\left(K_{0}\right)}\right)^{\frac{\varepsilon}{\varepsilon+d}}\left(\varepsilon^{s}\|v\|_{H^{2}\left(K_{0}\right)}\right)^{\frac{d}{\varepsilon+d}} .
$$

By using the fact that $\forall a, b \geq 0, \forall \rho \in[0,1], a^{\rho} b^{1-\rho} \leq a+b$, we obtain

$$
\|v\|_{H^{1}\left(K_{\varepsilon, r_{0}}\right)} \leq C\left(\mathrm{e}^{c / \varepsilon^{\mu}}\|P v\|_{L^{2}\left(K_{0}\right)}+\varepsilon^{s}\|v\|_{H^{2}\left(K_{0}\right)}\right) .
$$

We denote $I_{\varepsilon}=K_{\varepsilon} \cap \overline{B\left(x_{0}, r_{0}\right)}$, and $J_{\varepsilon}$ the complementary part of $I_{\varepsilon}$ in $b$ with $b=\Omega \cap B\left(x_{0}, r_{0}\right)$ (see the right-hand side of Fig. 2). Since for $x \in B\left(x_{0}, r_{0}\right)$ we have $\psi_{2}=d_{\partial \Omega}$, it is easy to verify that $I_{\varepsilon} \subset K_{\varepsilon, r_{0}}$. We finally have

$$
\|v\|_{H^{1}\left(I_{\varepsilon}\right)} \leq C\left(\mathrm{e}^{c / \varepsilon^{\mu}}\|P v\|_{L^{2}\left(K_{0}\right)}+\varepsilon^{s}\|v\|_{H^{2}\left(K_{0}\right)}\right) .
$$

The second step consists in finding an estimate of $\|v\|_{H^{1}\left(J_{\varepsilon}\right)}$ uniformly in $\varepsilon$, with the help of Lemma 2.4 in the domain $b$ for $v \in \tilde{H}_{0}^{2}(B)$. It follows that for all $\left.r \in\right] 0,1 / 2[$,

$$
\left\|\frac{v}{d_{\partial b}^{r}}\right\|_{L^{2}(b)} \leq C\|v\|_{H^{r}(b)}
$$

and since $d_{\partial b} \leq d_{\partial \Omega}=\psi_{2}<\varepsilon$ in $J_{\varepsilon}$

$$
\|v\|_{L^{2}\left(J_{\varepsilon}\right)} \leq C \varepsilon^{r}\|v\|_{H^{r}(b)} \leq C \varepsilon^{r}\|v\|_{H^{1 / 2}(b)}
$$

By using a classical interpolation inequality and a Young's inequality, it follows that $\forall \eta>0$,

$$
\|v\|_{L^{2}\left(J_{\varepsilon}\right)} \leq C^{\prime} \varepsilon^{r}\|v\|_{H^{1}(b)}^{1 / 2}\|v\|_{L^{2}(b)}^{1 / 2} \leq C^{\prime}\left(\frac{\varepsilon^{2 r}}{\eta}\|v\|_{H^{1}(b)}+\eta\|v\|_{L^{2}(b)}\right) .
$$

Since the above inequality is also true for the first derivatives of $v$, it follows that $\forall r \in] 0,1 / 2\left[, \exists C^{\prime}>0\right.$ such that $\forall \eta>0$,

$$
\|v\|_{H^{1}\left(J_{\varepsilon}\right)} \leq C^{\prime}\left(\frac{\varepsilon^{2 r}}{\eta}\|v\|_{H^{2}(b)}+\eta\|v\|_{H^{1}(b)}\right) .
$$

Using $\|v\|_{H^{1}(b)} \leq\|v\|_{H^{1}\left(I_{\varepsilon}\right)}+\|v\|_{H^{1}\left(J_{\varepsilon}\right)}$, and gathering (2.4) and (2.5), we obtain

$$
\|v\|_{H^{1}(b)} \leq C\left(\mathrm{e}^{c / \varepsilon^{\mu}}\|P v\|_{L^{2}\left(K_{0}\right)}+\varepsilon^{s}\|v\|_{H^{2}(B)}\right)+C^{\prime}\left(\frac{\varepsilon^{2 r}}{\eta}\|v\|_{H^{2}(B)}+\eta\|v\|_{H^{1}(b)}\right) .
$$


Choosing $s=2 r$ and $\eta$ such that $C^{\prime} \eta=1 / 2$, we obtain $\left.\forall r \in\right] 0,1 / 2[, \forall \mu>1, \exists c>0$ such that for sufficiently small $\varepsilon, \forall v \in \tilde{H}_{0}^{2}(B)$,

$$
\|v\|_{H^{1}(b)} \leq C\left(\mathrm{e}^{c / \varepsilon^{\mu}}\|P v\|_{L^{2}\left(K_{0}\right)}+\varepsilon^{2 r}\|v\|_{H^{2}(B)}\right),
$$

where $C$ is a new constant. We obtain that $\forall \kappa \in] 0,1\left[, \exists c>0\right.$ such that for sufficiently small $\varepsilon, \forall v \in \tilde{H}_{0}^{2}(B)$,

$$
\|v\|_{H^{1}(b)} \leq \mathrm{e}^{c / \varepsilon}\|P v\|_{L^{2}\left(K_{0}\right)}+\varepsilon^{\kappa}\|v\|_{H^{2}(B)} .
$$

The third step consists in coming back to a function $u \in H^{2}(\Omega)$. To this end we consider a function $\chi \in$ $C_{0}^{\infty}\left(B\left(x_{0}, R_{0}\right)\right)$ such that $\chi=1$ in $\overline{B\left(x_{0}, r_{1}\right)}$ with $0<r_{0}<r_{1}<R_{0}$, and $v=\chi u \in \tilde{H}_{0}^{2}(B)$. Applying the previous estimate to $v$, and denoting $D_{z, z^{\prime}}=B\left(x_{0}, z^{\prime}\right) \backslash \overline{B\left(x_{0}, z\right)}$ for $z<z^{\prime}$, one obtains there exists a new constant $C$ such that

$$
\|u\|_{H^{1}(b)} \leq C \mathrm{e}^{c / \varepsilon}\left(\|P u\|_{L^{2}\left(K_{0}\right)}+\|u\|_{H^{1}\left(K_{0} \cap D_{r_{1}, R_{0}}\right)}\right)+C \varepsilon^{\kappa}\|u\|_{H^{2}(B)} .
$$

Given the particular definition of $\psi_{2}$, we have $\overline{K_{0} \cap D_{r_{1}, R_{0}}} \subset \Omega$. Indeed, assume that $x \in \overline{K_{0} \cap D_{r_{1}, R_{0}}}$ and $d_{\partial \Omega}(x)=0$, then

$$
\psi_{2}(x)=-\frac{1}{2}(1-\gamma \circ r(x))\left|x-x_{0}\right|^{2} \leq-\frac{1}{2}\left(1-\gamma\left(r_{1}\right)\right) r_{1}^{2}<0,
$$

which is not possible. We conclude that there exists a neighborhood $\omega$ of $x_{0}$ and an open domain $\omega_{1} \Subset \Omega$ such that $\forall \kappa \in] 0,1\left[\right.$, there exist $\left.c, \varepsilon_{0}>0, \forall \varepsilon \in\right] 0, \varepsilon_{0}\left[, \forall u \in H^{2}(\Omega)\right.$,

$$
\|u\|_{H^{1}(\Omega \cap \omega)} \leq \mathrm{e}^{c / \varepsilon}\left(\|P u\|_{L^{2}(\Omega)}+\|u\|_{H^{1}\left(\omega_{1}\right)}\right)+\varepsilon^{\kappa}\|u\|_{H^{2}(\Omega)},
$$

which completes the proof.

Remark 2.3. It is natural to wonder if the proofs of Propositions 2.3 and 2.4 are still applicable to domains that are less smooth than $C^{1,1}$, in particular Lipschitz domains. The answer is clearly no. Indeed, as can be seen in the proof of our Carleman estimate in Proposition 2.1, the choice of $\psi_{1}$ and $\psi_{2}$ as set in (2.1)-(2.3) is not possible when $\Omega$ is not $C^{1,1}$ any longer, because in such situation (see Rem. 2.1) the components of $\nabla^{2} d_{\partial \Omega}$ and hence of $\nabla^{2} \psi_{i}(i=1,2)$ may be not functions any more in the classical sense. This is the reason why for Lipschitz domains, in particular, another technique has to be used (see [3]).

\subsection{Derivation of the final estimate}

Our final estimate for $C^{1,1}$ domains results from Propositions 2.2, 2.3 and 2.4. Precisely, Proposition 2.3 enables us to "propagate" Cauchy data on $\Gamma_{0}$ to a neighborhood of any smooth point $x_{0}$ of $\Gamma_{0}$, in particular to an open domain $\omega_{0} \Subset \Omega$. Proposition 2.2 enables us to "propagate" data from this open domain $\omega_{0}$ to any other open domain $\omega_{1} \Subset \Omega$. Lastly, Proposition 2.4 enables us to propagate data on an open domain $\omega_{1} \Subset \Omega$ up to a neighborhood of any point $x \in \partial \Omega$.

Theorem 2.2. Let $\Omega$ be a bounded and connected domain $\Omega \subset \mathbb{R}^{N}$ with a $C^{1,1}$ boundary $\partial \Omega$. If $\Gamma_{0}$ is a nonempty open domain of $\partial \Omega$, then

$\forall \kappa \in] 0,1\left[, \exists c, \varepsilon_{0}>0, \forall \varepsilon \in\right] 0, \varepsilon_{0}\left[, \forall u \in H^{2}(\Omega)\right.$,

$$
\|u\|_{H^{1}(\Omega)} \leq \mathrm{e}^{c / \varepsilon}\left(\|P u\|_{L^{2}(\Omega)}+\|u\|_{H^{1}\left(\Gamma_{0}\right)}+\left\|\partial_{n} u\right\|_{L^{2}\left(\Gamma_{0}\right)}\right)+\varepsilon^{\kappa}\|u\|_{H^{2}(\Omega)} .
$$

From Theorem 2.2 we obtain the following corollary.

Corollary 2.1. With the assumptions of Theorem $2.2, \forall \kappa \in] 0,1\left[, \exists C, \delta_{0}>0\right.$ such that $\left.\forall \delta \in\right] 0, \delta_{0}\left[, \forall u \in H^{2}(\Omega)\right.$ with

$$
\|u\|_{H^{2}(\Omega)} \leq M, \quad\|P u\|_{L^{2}(\Omega)}+\|u\|_{H^{1}\left(\Gamma_{0}\right)}+\left\|\partial_{n} u\right\|_{L^{2}\left(\Gamma_{0}\right)} \leq \delta,
$$


where $M$ is a constant,

$$
\|u\|_{H^{1}(\Omega)} \leq C \frac{M}{(\log (M / \delta))^{\kappa}} .
$$

Proof. We deduce from Theorem 2.2 that for $\varepsilon \leq \varepsilon_{0}$,

$$
\|u\|_{H^{1}(\Omega)} \leq \mathrm{e}^{c / \varepsilon} \delta+M \varepsilon^{\kappa}
$$

Denoting $f(\varepsilon)=\mathrm{e}^{c / \varepsilon} \delta+M \varepsilon^{\kappa}$ for $\varepsilon>0$, the minimizer $\varepsilon_{\min }$ of $f$ solves

$$
g\left(\varepsilon_{\min }\right)=\frac{M}{\delta}, \quad g(\varepsilon):=\frac{c}{\kappa} \frac{\mathrm{e}^{c / \varepsilon}}{\varepsilon^{\kappa+1}} .
$$

The function $g$ is non increasing with $g(0+)=+\infty$ and $g(+\infty)=0$, so that the above equation has a unique solution $\varepsilon_{\min }$ for each $\delta>0$.

If $\varepsilon_{0}>\varepsilon_{\min }$, then by choosing $\varepsilon=\varepsilon_{\min }$ in (2.7) we obtain that

$$
\|u\|_{H^{1}(\Omega)} \leq\left(\frac{\kappa}{c} \varepsilon_{0}+1\right) M \varepsilon_{\min }^{\kappa}=C M \varepsilon_{\min }^{\kappa} .
$$

For sufficiently small $\delta, \varepsilon_{\min }$ is sufficiently small to have for some $c^{\prime}>c$,

$$
\frac{M}{\delta}=g\left(\varepsilon_{\min }\right) \leq \mathrm{e}^{c^{\prime} / \varepsilon_{\min }}
$$

It follows that $\varepsilon_{\min } \leq c^{\prime} / \log (M / \delta)$, and we obtain the required result by plugging this estimate in (2.8). If $\varepsilon_{0} \leq \varepsilon_{\min }$, we obtain $g\left(\varepsilon_{0}\right) \geq M / \delta$, and thus

$$
\|u\|_{H^{1}(\Omega)} \leq M \leq g\left(\varepsilon_{0}\right) \delta=C \frac{M}{M / \delta}
$$

The result follows from the fact that for small $\delta, M / \delta \geq(\log (M / \delta))^{\kappa}$. In our proof, $C$ is independent of $u$, $M, \delta$.

Remark 2.4. Let $\Gamma_{1}$ denote the complementary part of $\Gamma_{0}$ in $\partial \Omega$. It follows from Corollary 2.1 that that for all $\kappa \in] 0,1[$,

$$
\|u\|_{H^{1 / 2}\left(\Gamma_{1}\right)}+\left\|\partial_{n} u\right\|_{H^{-1 / 2}\left(\Gamma_{1}\right)} \leq C(\kappa) \frac{M}{(\log (M / \delta))^{\kappa}},
$$

for all $u \in H^{2}(\Omega)$ such that $P u=0,\|u\|_{H^{2}(\Omega)} \leq M$ for some constant $M>0$ and $\|u\|_{H^{1}\left(\Gamma_{0}\right)}+\left\|\partial_{n} u\right\|_{L^{2}\left(\Gamma_{0}\right)} \leq \delta$ for sufficiently small $\delta$. This estimate should be compared to the one proved in [6] for $2 \mathrm{D}$ functions in $C^{2}(\bar{\Omega})$ with the help of a Carleman estimate obtained in [4].

It is useful to complete Theorem 2.2 with the following one in a truncated domain, which is more classical (see for example [13]). It results from Propositions 2.2 and 2.3.

Theorem 2.3. We consider a bounded and connected domain $\Omega \subset \mathbb{R}^{N}$ of class $C^{1,1}$. If $\Gamma_{0}$ is a non-empty open domain of $\partial \Omega$, then $\exists s, c, \varepsilon_{0}>0$ such that $\left.\forall \varepsilon \in\right] 0, \varepsilon_{0}\left[, \forall u \in H^{2}(\Omega)\right.$,

$$
\begin{gathered}
\|u\|_{H^{1}\left(\Omega_{\rho}\right)} \leq \frac{c}{\varepsilon}\left(\|P u\|_{L^{2}(\Omega)}+\|u\|_{H^{1}\left(\Gamma_{0}\right)}+\left\|\partial_{n} u\right\|_{L^{2}\left(\Gamma_{0}\right)}\right)+\varepsilon^{s}\|u\|_{H^{1}(\Omega)}, \\
\|u\|_{H^{2}\left(\Omega_{\rho}\right)} \leq \frac{c}{\varepsilon}\left(\|P u\|_{L^{2}(\Omega)}+\|u\|_{H^{3 / 2}\left(\Gamma_{0}\right)}+\left\|\partial_{n} u\right\|_{H^{1 / 2}\left(\Gamma_{0}\right)}\right)+\varepsilon^{s}\|u\|_{H^{1}(\Omega)},
\end{gathered}
$$

where $\Omega_{\rho}$ is defined, for small $\rho>0$, by $\Omega_{\rho}=\left\{x \in \Omega, d\left(x, \overline{\Gamma_{1}}\right)>\rho\right\}$, and $\Gamma_{1}$ is the open domain of $\partial \Omega$ such that $\Gamma_{0} \cap \Gamma_{1}=\emptyset$ and $\partial \Omega=\overline{\Gamma_{0}} \cup \overline{\Gamma_{1}}$. 
Proof. The estimate (2.9) is an obvious consequence of Propositions 2.2 and 2.3. The proof of (2.10) requires the following regularity estimate, which is easy to derive. For $\rho^{\prime}>\rho$, there exists $C>0$ such that for all $v \in H^{2}(\Omega)$ with $\left.v\right|_{\Gamma_{0}}=0$ and $\left.\left(\partial_{n} v\right)\right|_{\Gamma_{0}}=0$,

$$
\|v\|_{H^{2}\left(\Omega_{\rho^{\prime}}\right)} \leq C\left(\|v\|_{H^{1}\left(\Omega_{\rho}\right)}+\|P v\|_{L^{2}\left(\Omega_{\rho}\right)}\right)
$$

We can define $\left(\left.u\right|_{\Gamma_{0}},\left.\partial_{n} u\right|_{\Gamma_{0}}\right) \in H^{3 / 2}\left(\Gamma_{0}\right) \times H^{1 / 2}\left(\Gamma_{0}\right)$ for $u \in H^{2}(\Omega)$, and a continuous extension $E:\left(g_{0}, g_{1}\right) \in$ $H^{3 / 2}\left(\Gamma_{0}\right) \times H^{1 / 2}\left(\Gamma_{0}\right) \rightarrow \tilde{u} \in H^{2}(\Omega)$ such that $\left(\left.\tilde{u}\right|_{\Gamma_{0}},\left.\partial_{n} \tilde{u}\right|_{\Gamma_{0}}\right)=\left(g_{0}, g_{1}\right)$ (see [10], p. 37).

Let us suppose that $\tilde{u}=E\left(\left(\left.u\right|_{\Gamma_{0}},\left.\partial_{n} u\right|_{\Gamma_{0}}\right)\right)$. Since $v:=u-\tilde{u}$ satisfies $(2.9)$ with $\left.v\right|_{\Gamma_{0}}=0$ and $\left.\partial_{n} v\right|_{\Gamma_{0}}=0$, and since $v$ satisfies (2.11) as well, we obtain that for small $\rho>0$,

$$
\|v\|_{H^{2}\left(\Omega_{\rho}\right)} \leq \frac{c}{\varepsilon}\|P v\|_{L^{2}(\Omega)}+\varepsilon^{s}\|v\|_{H^{1}(\Omega)}
$$

We obtain the estimate (2.10) by coming back to the function $u$ and using the continuity of $E$.

\section{About the sharpness of the stability estimate}

In this section, we prove that the estimate (2.6) is nearly sharp in a sense we define later on. In this view, we take $P=-\Delta, \Omega$ is the $2 \mathrm{D}$ rectangle $] 0, X[\times] 0, Y\left[\right.$ and $\Gamma_{0}$ is the segment $] 0, Y[$ on the $y$ axis. $\Omega$ is not a domain of class $C^{1,1}$. Nevertheless, $(2.6)$ holds in $\Omega$ for functions $u$ defined in $] 0, X\left[\times \mathbb{R}\right.$ such that $u \in H^{2}(\Omega)$ and $u(x, y+Y)=u(x, y)$, for all $(x, y) \in] 0, X[\times \mathbb{R}$. We prove this simply by using Propositions $2.2,2.3,2.4$ and the $Y$-periodicity of function $u$ along the $y$ axis.

The estimate (2.6) is nearly sharp in the following sense: there does not exist a function $\varepsilon \rightarrow g(\varepsilon)$ with $\lim _{\varepsilon \rightarrow 0} g(\varepsilon) / \varepsilon=0$, such that for some $c, \varepsilon_{0}>0$, for all $\left.\varepsilon \in\right] 0, \varepsilon_{0}[$, for all $u$ such as described above,

$$
\|u\|_{H^{1}(\Omega)} \leq \mathrm{e}^{c / \varepsilon}\left(\|\Delta u\|_{L^{2}(\Omega)}+\|u\|_{H^{1}\left(\Gamma_{0}\right)}+\left\|\partial_{n} u\right\|_{L^{2}\left(\Gamma_{0}\right)}\right)+g(\varepsilon)\|u\|_{H^{2}(\Omega)}
$$

In other words, $g$ cannot decrease faster than $\varepsilon$ when $\varepsilon$ tends to 0 . Since in $(2.6) g(\varepsilon)=\varepsilon^{\kappa}$ for all $\kappa<1$, this proves that (2.6) is nearly sharp.

We prove this by contradiction. Assume $\lim _{\varepsilon \rightarrow 0} g(\varepsilon) / \varepsilon=0$. We define, for $X>0$ and $Y=2 \pi$, the following sequence of functions, which is inspired from the famous example of Hadamard

$$
u_{m}(x, y)=\phi(x) \mathrm{e}_{m}(x, y), \quad \mathrm{e}_{m}(x, y)=\mathrm{e}^{m x} \mathrm{e}^{i m y}
$$

with $m \in \mathbb{N}$ and $\phi$ is a $C^{2}$ function defined in $\mathbb{R}$ by

$$
\left\{\begin{array}{c}
\phi=0 \quad x \leq 0 \\
\phi \geq 0 \quad 0 \leq x \leq A \\
\phi=1 \quad x \geq A
\end{array}\right.
$$

with $X>A>0$.

We have of course $u_{m} \in H^{2}(\Omega), u_{m}(x, y+Y)=u_{m}(x, y)$ for all $\left.(x, y) \in\right] 0, X[\times \mathbb{R}$, and the definition of $\phi$ leads to $\left.u_{m}\right|_{\Gamma_{0}}=0$ and $\left.\left(\partial_{x} u_{m}\right)\right|_{\Gamma_{0}}=0$. From the stability estimate, we obtain that for all $m \in \mathbb{N}$ and for all $\varepsilon<\varepsilon_{0}$,

$$
\left\|u_{m}\right\|_{H^{1}(\Omega)} \leq \mathrm{e}^{c / \varepsilon}\left\|\Delta u_{m}\right\|_{L^{2}(\Omega)}+g(\varepsilon)\left\|u_{m}\right\|_{H^{2}(\Omega)}
$$


After some simple calculations, we have

$$
\begin{aligned}
\frac{\partial u_{m}}{\partial x} & =\left(m \phi+\phi^{\prime}\right) \mathrm{e}_{m}(x, y), \quad \frac{\partial u_{m}}{\partial y}=(i m \phi) \mathrm{e}_{m}(x, y), \\
\frac{\partial^{2} u_{m}}{\partial x^{2}} & =\left(m^{2} \phi+2 m \phi^{\prime}+\phi^{\prime \prime}\right) \mathrm{e}_{m}(x, y), \quad \frac{\partial^{2} u_{m}}{\partial x \partial y}=i m\left(m \phi+\phi^{\prime}\right) \mathrm{e}_{m}(x, y) \\
\frac{\partial^{2} u_{m}}{\partial y^{2}} & =-\left(m^{2} \phi\right) \mathrm{e}_{m}(x, y), \quad \Delta u_{m}=\left(2 m \phi^{\prime}+\phi^{\prime \prime}\right) \mathrm{e}_{m}(x, y) .
\end{aligned}
$$

Now let us consider the estimate (3.1). Concerning the left-hand side, we obtain after some simple calculations and by using the fact that $\phi(x)=1$ when $x \in[A, X]$ that

$$
\left\|u_{m}\right\|_{H^{1}(\Omega)} \geq C_{1} \sqrt{m} \sqrt{\mathrm{e}^{2 m X}-\mathrm{e}^{2 m A}}
$$

for some constant $C_{1}>0$. Concerning the right-hand side, by using the fact that $\sup _{i=0,1,2} \sup _{x \in \mathbb{R}}\left|\phi^{(i)}(x)\right|<$ $+\infty$ and $\phi^{\prime}(x)=0$ when $x \in[A, X]$,

$$
\left\|\Delta u_{m}\right\|_{L^{2}(\Omega)} \leq C_{2} \sqrt{m} \sqrt{\mathrm{e}^{2 m A}-1}, \quad\left\|u_{m}\right\|_{H^{2}(\Omega)} \leq C_{3} m^{3 / 2} \sqrt{\mathrm{e}^{2 m X}-1},
$$

for some constants $C_{2}, C_{3}>0$. Combining the estimates (3.1), (3.2) and (3.3), we obtain that for all $m$ and all $\varepsilon<\varepsilon_{0}$,

$$
\sqrt{\mathrm{e}^{2 m X}-\mathrm{e}^{2 m A}} \leq C \mathrm{e}^{c / \varepsilon} \sqrt{\mathrm{e}^{2 m A}-1}+C g(\varepsilon) m \sqrt{\mathrm{e}^{2 m X}-1},
$$

for some constant $C>0$. Dividing the above equation by $\sqrt{\mathrm{e}^{2 m X}-1}$, we obtain

$$
\frac{\sqrt{1-\mathrm{e}^{-2 m(X-A)}}}{\sqrt{1-\mathrm{e}^{-2 m X}}} \leq C \mathrm{e}^{c / \varepsilon} \mathrm{e}^{-m(X-A)} \frac{\sqrt{1-\mathrm{e}^{-2 m A}}}{\sqrt{1-\mathrm{e}^{-2 m X}}}+C g(\varepsilon) m \text {. }
$$

It remains to select $\eta$ such that $0<\eta<X-A$ and define the sequence $\left(\varepsilon_{m}\right)_{m}$ such that $\varepsilon_{m}=1 /(k m)$ with $k=(X-A-\eta) / c>0$. Hence we have $\mathrm{e}^{c / \varepsilon_{m}-m(X-A)}=\mathrm{e}^{-\eta m}$. The left-hand side of (3.4) converges to 1 when $m \rightarrow+\infty$, while the first term of the right-hand side tends to 0 when $\varepsilon$ is replaced by $\varepsilon_{m}$, as well as the second term since $g(1 / m) m \rightarrow 0$ when $m \rightarrow+\infty$. Thus, we have found a contradiction.

Remark 3.1. To the author's knowledge, the validity of (2.6) in the limit case $\kappa=1$ is an open problem, even for domains of class $C^{\infty}$.

\section{Application to the method of QUASI-REVERSIBILITy}

In this section, we use the stability estimates obtained before in order to derive some convergence rates for the quasi-reversibility method, and therefore to complete the results already obtained in [16] in truncated domains. The method of quasi-reversibility, first introduced in [17], enables one to regularize the ill-posed elliptic Cauchy problems. Specifically, we consider a domain $\Omega$ as described in the statement of Theorem 2.2, and a truncated domain $\Omega_{\rho}$ as defined in the statement of Theorem 2.3.

Now we assume that $u \in H^{2}(\Omega)$ solves the ill-posed Cauchy problem with $\left(g_{0}, g_{1}\right) \in H^{3 / 2}\left(\Gamma_{0}\right) \times H^{1 / 2}\left(\Gamma_{0}\right)$ :

$$
\left\{\begin{array}{c}
P u=0 \text { in } \Omega \\
\left.u\right|_{\Gamma_{0}}=g_{0} \\
\left.\partial_{n} u\right|_{\Gamma_{0}}=g_{1} .
\end{array}\right.
$$


Given some noisy data $\left(g_{0}^{\sigma}, g_{0}^{\sigma}\right) \in H^{3 / 2}\left(\Gamma_{0}\right) \times H^{1 / 2}\left(\Gamma_{0}\right)$ with

$$
\left\|g_{0}^{\sigma}-g_{0}\right\|_{H^{3 / 2}\left(\Gamma_{0}\right)}+\left\|g_{1}^{\sigma}-g_{1}\right\|_{H^{1 / 2}\left(\Gamma_{0}\right)} \leq \sigma
$$

we consider the formulation of quasi-reversibility for $\alpha>0$ : find $u_{\alpha}^{\sigma} \in H^{2}(\Omega)$, such that $\forall v \in H^{2}(\Omega),\left.v\right|_{\Gamma_{0}}=$ $\left.\partial_{n} v\right|_{\Gamma_{0}}=0$,

$$
\left\{\begin{array}{c}
\left(P u_{\alpha}^{\sigma}, P v\right)_{L^{2}(\Omega)}+\alpha\left(u_{\alpha}^{\sigma}, v\right)_{H^{2}(\Omega)}=0 \\
\left.u_{\alpha}^{\sigma}\right|_{\Gamma_{0}}=g_{0}^{\sigma} \\
\left.\partial_{n} u_{\alpha}^{\sigma}\right|_{\Gamma_{0}}=g_{1}^{\sigma} .
\end{array}\right.
$$

Using Lax-Milgram theorem, we easily prove that formulation (4.2) is well-posed. If we denote $u_{\alpha}=u_{\alpha}^{0}$, which is the solution of quasi-reversibility without noise, we obtain for some constant $C_{0}>0$,

$$
\left\|u_{\alpha}^{\sigma}-u_{\alpha}\right\|_{H^{2}(\Omega)} \leq C_{0} \frac{\sigma}{\sqrt{\alpha}}
$$

On the other hand, we easily prove by using (4.1) and (4.2) that there exist constants $C_{1}, C_{2}>0$ such that

$$
\left\|u_{\alpha}-u\right\|_{H^{2}(\Omega)} \leq C_{1}, \quad\left\|P\left(u_{\alpha}-u\right)\right\|_{L^{2}(\Omega)} \leq C_{2} \sqrt{\alpha} .
$$

Using (4.4) and then Corollary 2.1, Theorem 2.3 (combined with Lem. 2.3) for function $u_{\alpha}-u \in H^{2}(\Omega)$, we obtain there exist $\gamma \in] 0,1 / 2[, C(\kappa)>0$ for all $\kappa \in] 0,1[$, such that for sufficiently small $\alpha>0$,

$$
\begin{gathered}
\left\|u_{\alpha}-u\right\|_{H^{2}\left(\Omega_{\rho}\right)} \leq C \alpha^{\gamma} \\
\left\|u_{\alpha}-u\right\|_{H^{1}(\Omega)} \leq C(\kappa) \frac{1}{(\log (1 / \alpha))^{\kappa}} .
\end{gathered}
$$

Choosing $\alpha=\sigma$ in (4.3), we obtain exactly the same estimates for $u_{\alpha}^{\sigma}-u$ as in (4.5) and (4.6) simply by replacing the regularization parameter $\alpha$ by the amplitude of noise $\sigma$ in the right-hand side.

Remark 4.1. In [2], Theorem 3 is not optimal in the sense that we can obtain the Hölder convergence rate (4.5) and not only a logarithmic convergence rate as stated in the theorem.

Remark 4.2. It should be easy to prove Theorem 2.2 and Corollary 2.1 for some more general elliptic operators, for example

$$
P u=-\sum_{i, j=1}^{N} \partial_{i}\left(a_{i j}(x) \partial_{j} u\right)+\sum_{j=1}^{N} b_{j}(x) \partial_{j} u+c(x) u,
$$

if we assume that the coefficients $b_{j}(j=1, \ldots, N)$ and $c$ all belong to $L^{\infty}(\Omega)$, that the coefficients $a_{i j}$ are Lipschitz continuous in $\bar{\Omega}$, satisfy $a_{i j}=a_{j i}$ and for some constant $a>0$,

$$
\sum_{i, j=1}^{N} a_{i j}(x) \xi_{i} \xi_{j} \geq a|\xi|^{2}, \quad \forall x \in \bar{\Omega}, \quad \forall \xi \in \mathbb{R}^{N} .
$$

Remark 4.3. There is an analogy of our result with case of the stability estimate for the parabolic equation with the reversed time direction, that is the problem of finding the initial condition from the lateral Cauchy data in the time interval $] 0, T[$. One obtains for that problem a Hölder stability estimate of the solution in the incomplete time interval ] $T$ [ with $a>0$ [18] and one obtains a logarithmic stability estimate when $a=0$ [15].

Acknowledgements. The author thanks M.V. Klibanov for being at the origin of Remark 4.1 and J. Le Rousseau for his lesson in global Carleman estimates. He also thanks the referees for their valuable comments. 


\section{REFERENCES}

[1] G. Alessandrini, E. Beretta, E. Rosset and S. Vessella, Optimal stability for inverse elliptic boundary value problems with unknown boundaries. Ann. Scuola Norm. Sup. Pisa 29 (2000) 755-806.

[2] L. Bourgeois, Convergence rates for the quasi-reversibility method to solve the Cauchy problem for Laplace's equation. Inv. Prob. 22 (2006) 413-430.

[3] L. Bourgeois and J. Dardé, Conditional stability for ill-posed elliptic Cauchy problems: the case of Lipschitz domains (part II). Rapport INRIA 6588, France (2008).

[4] A.L. Bukhgeim, Extension of solutions of elliptic equations from discrete sets. J. Inv. Ill-Posed Problems 1 (1993) 17-32.

[5] T. Carleman, Sur un problème d'unicité pour les systèmes d'équations aux dérivées partielles à deux variables indépendantes. Ark. Mat. Astr. Fys. 26 (1939) 1-9.

[6] J. Cheng, M Choulli and J. Lin, Stable determination of a boundary coefficient in an elliptic equation. M3AS 18 (2008) $107-123$.

[7] M.C. Delfour and J.-P. Zolésio, Shapes and geometries. SIAM, USA (2001).

[8] C. Fabre and G. Lebeau, Prolongement unique des solutions de l'équation de Stokes. Comm. Part. Differ. Equ. 21 (1996) $573-596$.

[9] A. Fursikov and O. Imanuvilov, Controllability of Evolution Equations, Lecture Notes Series 34. Research Institute of Mathematics, Seoul National University, South Korea (1996).

[10] P. Grisvard, Elliptic problems in nonsmooth domains. Pitman, USA (1985).

[11] L. Hormander, Linear Partial Differential Operators. Fourth Printing, Springer-Verlag, Germany (1976).

[12] T. Hrycak and V. Isakov, Increased stability in the continuation of solutions to the Helmholtz equation. Inv. Prob. 20 (2004) $697-712$.

[13] V. Isakov, Inverse problems for partial differential equations. Springer-Verlag, Berlin, Germany (1998).

[14] F. John, Continuous dependence on data for solutions of pde with a prescribed bound. Commun. Pure Appl. Math. 13 (1960) $551-585$.

[15] M.V. Klibanov, Estimates of initial conditions of parabolic equations and inequalities via lateral data. Inv. Prob. 22 (2006) 495-514.

[16] M.V. Klibanov and A.A. Timonov, Carleman Estimates for Coefficient Inverse Problems and Numerical Applications. VSP (2004).

[17] R. Lattès and J.-L. Lions, Méthode de quasi-réversibilité et applications. Dunod, France (1967).

[18] M.M. Lavrentiev, V.G. Romanov and S.P. Shishatskii, Ill-posed problems in mathematical physics and analysis. Amer. Math. Soc., Providence, USA (1986).

[19] G. Lebeau and L. Robbiano, Contrôle exact de l'équation de la chaleur. Commun. Partial Differ. Equ. 20 (1995) $335-356$.

[20] L.E. Payne, On a priori bounds in the Cauchy problem for elliptic equations. SIAM J. Math. Anal. 1 (1970) 82-89.

[21] K.-D. Phung, Remarques sur l'observabilité pour l'équation de Laplace. ESAIM: COCV 9 (2003) 621-635.

[22] L. Robbiano, Théorème d'unicité adapté au contrôle des solutions des problèmes hyperboliques. Commun. Partial Differ. Equ. 16 (1991) 789-800.

[23] D.A. Subbarayappa and V. Isakov, On increased stability in the continuation of the Helmholtz equation. Inv. Prob. 23 (2007) $1689-1697$.

[24] T. Takeuchi and M. Yamamoto, Tikhonov regularization by a reproducing kernel Hilbert space for the Cauchy problem for a elliptic equation. SIAM J. Sci. Comput. 31 (2008) 112-142. 